\title{
Jensen-Shannon Complexity and Permutation Entropy Analysis of Geomagnetic Auroral Currents
}

\author{
Adnane Osmane ${ }^{1}$, Andrew P. Dimmock ${ }^{2}$, Tuija I. Pulkkinen ${ }^{3,4}$ \\ ${ }^{1}$ Department of Physics, University of Helsinki, Helsinki, Finland \\ ${ }^{2}$ Swedish Institute of Space Physics, Uppsala University, Uppsala, Sweden \\ ${ }^{3}$ Department of Climate and Space Sciences, University of Michigan, Ann Arbor, MI, USA \\ ${ }^{4}$ Aalto University, School of Electrical Engineering, Espoo, Finland
}

\section{Key Points:}

- Jensen-Shannon complexity plane used to analyse auroral geomagnetic indices.

- Auroral indices are shown to be inconsistent with low-dimensional chaotic processes.

- Maximum in complexity occurs on timescales ranging between 10 and 40 minutes.

This is the author manuscript accepted for publication and has undergone full peer review but has not been through the copyediting, typesetting, pagination and proofreading process, which may lead to differences between this version and the Version of Record. Please cite this article as doi: 10.1029/2018JA026248

Corresponding author: Adnane Osmane, adnane.osmane@helsinki.fi

This article is protected by copyright. All rights reserved. 


\begin{abstract}
In this study we determine whether auroral westward currents can be characterised by low dimensional chaotic attractors through the use of the complexity-entropy methodology developed by Rosso, Larrondo, Martin, Plastino, and Fuentes (2007) and based on the permutation entropy developed by Bandt and Pompe (2002). Our results indicate that geomagnetic auroral indices are indistinguishable from stochastic processes from timescales ranging from a few minutes to 10 hours and for embedded dimensions $d<$ 8. Our results are inconsistent with earlier studies of (Baker, Klimas, McPherron, \& Büchner, 1990; Pavlos et al., 1992; D. Roberts, Baker, Klimas, \& Bargatze, 1991; D. A. Roberts, 1991; Vassiliadis, Sharma, \& Papadopoulos, 1991; Vassiliadis, Sharma, Eastman, \& Papadopoulos, 1990) indicating that auroral geomagnetic indices could be reduced to lowdimensional systems with chaotic dynamics.
\end{abstract}

\title{
1 Introduction
}

The discovery fifty years ago that fully developed turbulence could in principle be the result of only three instabilities (Ruelle \& Takens, 1971), rather than an infinite number Landau (1944), together with the experimental confirmation by Gollub and Swinney (1975) that universal behavior described by a few parameters could be observed in a fluid system, has lead to what some authors described as a "chaos revolution" (Lovejoy \& Schertzer, 1998). The realisation that nonlinear systems with a very large number of degree of freedoms could be described by low-dimensional dynamical systems naturally found a promising niche in a wide range of space plasma research, and especially in space weather studies, in order to alleviate the computational cost of modelling the Earth's magnetosphere. Following the development of empirical techniques for detecting deterministic chaos by Grassberger and Procaccia (1983), a plethora of studies (Baker et al., 1990; Pavlos et al., 1992; D. Roberts et al., 1991; D. A. Roberts, 1991; Vassiliadis et al., 1991; Vassiliadis et al., 1990) using geomagnetic indices argued that the Earth's magnetospheric dynamics could be reduced to a low-dimensional dynamical systems. However, it was first shown by Osborne and Provenzale (1989) for a general case, and later by Prichard and Price (1992) and Shan, Hansen, Goertz, and Smith (1991) for space weather studies, that the empirical technique could not differentiate between coloured noise and deterministic chaos in geomagnetic time series, due to long autocorrelation times inherent to the former. 
Nonetheless, the ideas provided by deterministic chaos were extended to nonlinear stochastic systems by the use of self-organized critical (SOC) models, that is cellular automata defined by a certain class of discontinuous rules and appropriate boundary conditions (Lovejoy \& Schertzer, 1998). SOC systems were shown to evolve spontaneously to critical states describable by a low-dimensional dynamical systems (Chang, 1992). Consequently, Balasis et al. (2006); Consolini and Marcucci (1997); Dobias and Wanliss (2009); Klimas et al. (2000); Klimas, Vassiliadis, Baker, and Roberts (1996); A. Pulkkinen, Klimas, Vassiliadis, and Uritsky (2006); Uritsky, Pudovkin, and Steen (2001); Uritsky, Klimas, and Vassiliadis (2006); Uritsky and Pudovkin (1998); Valdivia, Klimas, Vassiliadis, Uritsky, and Takalo (2003); Wanliss, Anh, Yu, and Watson (2005); Wanliss and Dobias (2007) extended these ideas to magnetospheric systems, demonstrating that nonlinear stochastic models were a better representation than low-dimensional chaotic attractors. The emergence of dynamical correlations and non-Markovian features during intense geomagnetic storms, analogous to the emergence of long-range coherence in out-of-equilibrium systems, implied a reduction in the number of degrees of freedom of the system and inherent nonlinearities (Consolini \& De Michelis, 2014).

In this study, we contribute to the decades old discussions on the properties of geomagnetic processes by using permutation entropy, a measure developed by Bandt and Pompe (2002), to quantify complexity in measured time series. Whereas common measures of complexity, such as the Kolmogorov-Sinai entropy, or the Shannon entropy, ignore temporal order of the values in the time series, entropy measures of ordinal patterns preserve information of temporal order and provides for an alternative measure of complexity (Riedl, Muller, \& Wessel, 2013). Permutation entropy has now been tested across several scientific disciplines, and is now being used to characterise processes in laboratory and geophysical plasma experiments (Consolini \& De Michelis, 2014; Maggs \& Morales, 2013; Weck, Schaffner, Brown, \& Wicks, 2015).

For example, using the complexity-entropy measure developed by Rosso et al. (2007), Weck et al. (2015) demonstrate that solar wind turbulent fluctuations are stochastic, rather than chaotic. In the context of geomagnetic activity, it is of primary interest to determine whether, and under which time scales, geomagnetic currents demonstrate signatures of low-dimensional dynamical systems, if any. Using the methodology developed by Rosso et al. (2007), we hereafter revisit the question as to whether auroral geomagnetic indices can be characterised as a low-dimensional chaotic attractor, and use for the 
first time the Jensen-Shannon complexity on auroral geomagnetic indices. In section 2 we describe the dataset and the complexity-entropy plane used for distinguishing between stochastic and chaotic time series. In section 3 we present the results. In section 4 and 5 we discuss our findings, their relation to previous studies, and trace out a plan for future studies.

\section{Methodology}

\subsection{Datasets}

The data are obtained from the OMNI database (http://omniweb.gsfc.nasa.gov), which provides estimates of solar wind parameters at the bow shock nose (Farris \& Russell, 1994) by propagating observations performed by several spacecraft further upstream (King \& Papitashvili, 2005) as well as measures of geomagnetic activity. We focus primarily on the $A L$ index which is notoriously difficult to predict Newell, Sotirelis, Liou, Meng, and Rich (2007), presumably because of inherent nonlinearities in its dynamics.

$A L$ provides an estimate of the maximum westward electrojet intensity using 12 magnetometer stations around the northern auroral region (Berthelier \& Menvielle, 1993). Outside of substorm intervals, AL can be thought of as a measure of convection, while during substorms the largest deviations in the horizontal component typically originate from the substorm current wedge. By nature, AL is therefore highly asymmetric and peaks at low values, reflecting quiet time convection effects and heavy tails associated with substorms occurrences (Newell et al., 2007; Tanskanen, Pulkkinen, Koskinen, \& Slavin, 2002).

\subsection{Permutation Entropy}

Permutation entropy was proposed by Bandt and Pompe (2002) as a complexity measure for arbitrary timeseries, that is, stationary or non-stationary, deterministic or stochastic, periodical or noisy. However, it should be pointed that a weak form of stationary assumption is required, i.e., for $s<d$, the probability for $x_{t}<x_{t+s}$ should not depend on $t$ (Rosso et al., 2013). The Bandt-Pompe permutation entropy is computed on the basis of a probability distribution quantifying the rate of occurrence of amplitude orderings in a time signal $\mathcal{T}(t) \equiv\left\{x_{t} ; t=1, \ldots, N\right\}$ measured at $N$ evenly spaced discrete points. Computation of the probability is done for an embedding space of dimension $d$, which translates in determining patterns of length $d$ in the order in which 
they appear in the timeseries (For readers not familiar with the idea of an embedding dimension and delay we recommend the book by (Ott, 2002, Section 3.8)). The $d$-values are called $d$-tuples. For instance, for $d=3$, a number of $d !=6$ possible sequences are possible, i.e. $(1,2,3),(1,3,2),(2,1,3),(2,3,1),(3,1,2)$ and $(3,2,1)$. For a signal with $N$ elements, the relative frequency of each of the possible sequences are computed for three successive values of the time series. The number of successive values for embedding dimension $d$ and signal of $N$ elements consists in $1 \leq n \leq N-d+1$ distinct d-tuples ordered as $t_{j}, t_{j+1} \ldots t_{j+d-1}$. Similarly, for the embedding dimension chosen in our analysis, i.e. $d=6$, a signal with $N=10000$ elements has $1 \leq n \leq N-d+1=9995$ distinct d-tuples $t_{j}, t_{j+1} \ldots t_{j+5}$, or ordinal patterns. Within each d-tuplet, an ordering of the amplitude is obtained as a function of the $d$ ! possible permutations, e.g. 720 permutations for $d=6$. The permutation entropy is then computed for a particular signal by computing the frequency of occurrence of each possible permutations of the amplitude ordering. For a set of probabilities $P$, of dimension $d$ ! and probability of occurrence $p_{j} \geq 0 ; j=1,2 \ldots d !$, the permutation entropy is defined in terms of the Shannon entropy, $S$, as

$$
S(P)=-\sum_{j=1}^{d !} p_{j} \log \left(p_{j}\right) .
$$

In the following we use the normalized Shannon entropy, $H$, defined as

$$
H(P)=S(P) / \log (d !)=S(P) / P_{e},
$$

and the Shannon entropy per symbol, $h_{n}$, defined as

$$
h_{n}(P)=\frac{S(P)}{d-1} .
$$

The denominator in the equation of $H(P)$ is the maximum Shannon entropy obtained when all states have equal probabilities, i.e. $p_{j}=1 / d ! ; \forall j$ and this maximum probability is here denoted as $P_{e}$. The fundamental underlying idea behind the Bandt-Pompe permutation entropy is that some ordinal patterns may be forbidden, whereas others may be favoured, making the information content less random than in stochastic systems. In theory, one might therefore be able to differentiate stochastic and deterministic fluctuations through the use of the permutation entropy.

However, as with all mathematical tools, one has to be aware of the advantages and limitations. In terms of advantages, the permutation entropy incorporates temporal order and is computationally very fast (Riedl et al., 2013). Additionally it is invariant under 
any monotonic transformation of the timeseries, e.g. scaling the data has no effect on the resulting distribution of permutation patterns. A consequence of the latter property is that the permutation entropy does not preserve information of the amplitude in the ordinal patterns. But more importantly, finite timeseries constrain the choice of the embedding dimension $d$. Since the number of possible amplitude permutations increases rapidly as $d$ !, the value of $d$ must be chosen such that $N \gg d$ !, i.e. the number of points in our time series must be sufficiently large for us to sample the relative distribution of each $d$ ! permutations. In our case, we use $d=6$ for timeseries with $N=43200$ points, corresponding to 30 days of 1 minute sampling. The maximum embedded delay corresponds to $\tau=600$ minutes, and the minimum number of segments corresponds to $N-(d-$ 1) $\tau>40000$ (Applying our analysis to delays ranging from days to months none of the conclusions presented hereafter were modified. However it was not possible to test the methodology for timescales ranging from years to solar cycle periods due to data gaps). Hence, we use more than 40000 segments to distinguish the frequency of $d !=720$ patterns. Consequently, for a given collection of $d$-tuplets, the size in time of the structures, or patterns, investigated is $d \Delta t$, where $\Delta t$ is the sampling time. In order to study structures with size $d \Delta t \geq 10$ it is often not practically possible to increase the embedding dimension beyond $d=7$ since $8 !=40320$ and one must keep in mind the requirement that $N \gg d$ !. In case of auroral indices, setting an embedding dimension of 8 would require a timeseries of length $N>4 \cdot 10^{6}$, and corresponding to 280 days of 1 minute sampled data. Large structures can nonetheless still be investigated by adding an additional parameter $\tau$ to sub-sample the timeseries. In the sub-sampled signal the interval between successive data point is $d \tau$ rather $\Delta t$. This technique naturally reduces the Nyquist frequency and the number of points to $N / \tau$, but preserves the total time of the signal (Maggs \& Morales, 2013; Weck et al., 2015). For an embedding dimension $d>$ 2 and embedded delay $\tau$, a timeseries with $N$ points contains $N-(d-1) \tau$ segments upon which the $d$ ! permutations are computed. Once again, one needs to be very careful in selecting a sufficiently large number of points to make sure that all possible permutations can be accounted for. If one only has 1000 segments available to sample the relative occurrence of 720 permutations, it is highly unlikely that even if the timeseries is stochastic, that all possible permutations would be appropriately sampled. One might therefore conclude, incorrectly, that some patterns are forbidden, and that the resulting entropy might be indicative of a deterministic timeseries. The timeseries length must 
be sufficiently large in order for all permutation patterns to be measured and thereby confirm the deterministic properties of the timeseries. This last constraint is particularly important when studying coloured noise with very long autocorrelation times. The permutation entropy analysis can be rendered useless if $N$ is not sufficiently large and one is therefore forced to seek alternative approaches to differentiate stochastic from deterministic fluctuations.

\subsection{Jensen-Shannon Complexity}

A solution to supersede this last limitation of the permutation entropy and a means to distinguish between long correlated noise and deterministic timeseries, was outlined by Rosso et al. (2007). Using Shannon's formulation of entropy for ordinal patterns and the Jensen-Shannon complexity as a measure of statistical complexity, Rosso et al. (2007) have shown that despite common properties (wide-band power spectrum, irregular behavior of measured signals) it is after all possible to distinguish between stochastic and chaotic signals from their location in terms of an entropy-complexity plane. Hence, Rosso et al. (2007) combine the complexity measure of Bandt and Pompe and the Jensen-Shannon complexity, here defined as

$$
C_{J}^{S}=D(P) \times H(P)=-2 \frac{S\left(\frac{P+P_{e}}{2}\right)-\frac{1}{2} S(P)-\frac{1}{2} S\left(P_{e}\right)}{\frac{d !+1}{d !} \log (d !+1)-2 \log (2 d !)+\log (d !)} H(P) .
$$

This complexity measure is the product of the normalised Shannon entropy, $H(P)$, and the Jensen divergence,

$$
D(P)=S\left(\frac{P+P_{e}}{2}\right)-\frac{1}{2} S(P)-\frac{1}{2} S\left(P_{e}\right),
$$

hence the Jensen-Shannon denomination. The argument in the denominator serves as normalisation constant for the Jensen divergence. The divergence can be interpreted as the distance between our distribution of ordinal patterns and the distribution that maximises the Shannon distribution, i.e. $P_{e}$ defined above. It is easy to see that it takes the value of zero when $P=P_{e}$, that is, when all ordinal patterns are equally likely the JensenShannon complexity is zero. Built from the square of the Shannon entropy, it has a parabolic shape when plotted against the permutation entropy, but more crucially, the Jensen-Shannon complexity can hold multiple values for a fixed Shannon entropy. It is this particular property that allows one to distinguish stochastic noise with long auto-correlation times to deterministic and chaotic fluctuations. Thus, a fixed entropy value maps into a range of Jensen-Shannon complexity values and one can differentiate between regimes that are 
highly deterministic or highly stochastic and everything in-between. For more details on the permutation entropy and Jensen-Shannon complexity we refer to the reviews of Riedl et al. (2013) and Zanin, Zunino, Rosso, and Papo (2012).

\section{Results}

\subsection{Choice of chaotic and stochastic timeseries}

In this study we benchmark our results for geomagnetic indices with the Lorenz chaotic attractor (Ott, 2002) :

$$
\dot{X}=a(Y-X) ; \quad \dot{Y}=X(c-Z)-Y ; \quad \dot{Z}=X Y-b Z,
$$

and fractional Brown motion (fBm) with Hurst exponent $h_{u} \in[0.01,1]$ (Mandelbrot \& Van Ness, 1968). We note that the choice of the Lorenz attractor rather than other well-known chaotic dynamical systems has no effect on our results. The reader can find a longer list of chaotic maps plotted in the complexity-entropy plane in reports by Rosso et al. (2007) and Maggs and Morales (2013) showing a clear demarcation between stochastic and chaotic timeseries. The parameters for the Lorenz attractor are $a=10, b=8 / 3, c=$ 28. Figure 1 top two panels show the timeseries for $A L$ in black, and $\delta A L=A L_{j+1}-$ $A L_{j}$ in magenta for four months time interval.Time series for the Lorenz strange attractor used for this study are shown as blue traces in Figure 1 and an example for the fBm with Hurst exponent $h_{u}=0.8$ is shown on the bottom right panel of Figure 1 in red. The $\mathrm{fBm}$ is stochastic but can nonetheless be structured and contains trends (either persistent or anti-persistent), and is used as the boundary delimiting stochastic and chaotic timeseries.

\subsection{Permutation entropy analysis}

In Figure 2, we plot the permutation entropy per symbol, $h_{n}$, for a stochastic, a chaotic and the AL time series as a function of embedded delay $\tau$ and embedded dimension $3 \leq$ $d \leq 7$. Fractional Brownian motion with Hurst exponent of 0.75 is on the top panel. As with very other stochastic timeseries the permutation entropy is approximately constant across various $\tau$ and $d$. The small increase in the entropy is due to the fact tfhat our choice of stochastic process has long auto-correlation times. Thus, for very long time delays, patterns become marginally more decorrelated. 
In contrast, the centre panel for the chaotic Lorenz attractor shows a minimum permutation entropy for $\tau=1$. The permutation entropy then increases linearly with $\tau$, until for sufficiently large delay of $\tau>15$ the patterns become decorrelated. We also notice from the Lorenz attractor panel that for $\tau<10$ the curves for $d=7$ and $d=6$ overlap. Hence, embedded dimension $d=6$ is sufficient to track all the possible permutations for the Lorenz attractor. Or, put differently, increasing the embedded dimension to $d>6$ does not provide more information about the complexity of the timeseries. Increasing the embedding dimension increases the range of patterns that are sampled in the timeseries. If one is analyzing a dynamical system with embedding dimension of 6 or 7 with a parameter $d=3-4$, one will miss some of the possible permutations. Thus, the result plotted for the Lorenz attractor is not surprising, since according to the well-known Takens' theorem (Ott, 2002), the embedded dimension must scale as $d=$ $2 d_{S}+1$ where $d_{S}$ is the dimension of the strange attractor, which is well-known to be between 2.03 and 2.06 .

It is clear that the profile for $A L$, in the bottom panel, resembles the stochastic fractional Brownian motion with persistent increments. However, the absence of overlap for the various curves, as seen for the Lorenz attractor, does not necessarily imply that $A L$ is stochastic. Instead, it could indicate that a higher embedded dimension, i.e. $d>7$, is needed to samples all the patterns (Rosso et al., 2013). However, it is not always possible to pick $d>7$, and we resort to the methodology of Rosso et al. (2007) to determine possible differences between $A L$ and stochastic fluctuations.

\subsection{Jensen-Shannon complexity plane}

Before making use of the complexity-entropy plane, we first present the Jensen-Shannon complexity measure for $A L$ as a function of embedded dimension and delay. The results are shown in Figure 3. The Jensen-Shannon complexity is computed against the embedded delay $\tau$ on the abscissa for all twelve months of the year 2010. The colour represents the embedding dimension ranging between 3 and 6 with the same legend as in Figure 2. Note that the complexity decreases for growing embedded delay. Hence, ordinal patterns are more correlated on small timescales $(\tau<1$ hour), and become decorrelated for $\tau>200-240$ minutes. Since the embedding dimension of $A L$ is high, the complexity curves for $d=6$ and $d=5$ highlights local maximum and minimum that are missed by the $d=4$ and $d=3$ curve. For all months except May, June, July and Novem- 
ber, we notice an enhancement in the complexity for $14<\tau<40$ and $d=6$ after the initial monotonic decrease in complexity. A local maximum in complexity is particularly pronounced for the months of August and September. This indication of the presence of correlational structures with timescales ranging between 10 and 40 minutes is not a new result (Osmane, Dimmock, Naderpour, Pulkkinen, \& Nykyri, 2015) and will be discussed in the next section.

In Figure 4, we plot the complexity-entropy plane for $d=6$, with minimum and maximum complexity-entropy curves in blue. The complexity-entropy points for fBm are plotted in red circles for $\tau=1$ and Hurst exponents ranging between 0.01 and 1 by steps of 0.01 . The points for $\mathrm{fBm}$ indicate a limit between stochastic and structured timeseries. The complexity-entropy values for the chaotic attractor are computed and plotted in blue triangles for the variable $X$, for $d=6$ and $\tau=[1-10]$. The complexity-values for the Lorentz attractor (cyan squares) have the smallest entropy for $\tau=1$ and the largest for $\tau=60$. We clearly see from Figure 4 that the complexity-entropy values for the Lorenz chaotic orbits skim the maximum curve, that is for large entropies, the orbits of a chaotic attractor have large complexity values.

Similarly, we plot on the same figure the complexity-entropy curve for both $A L$ (black stars) and $\delta A L=\operatorname{diff}(A L)$ (magenta dots) for $d=6$ and time delay values ranging between $\tau=4$ and $\tau=600$ minutes by increments of 2 minutes. The lowest entropy values for $A L$ and $\delta A L$ are computed for $\tau=4$, while the larger entropy values are for large $\tau>500$. Figure 4 indicates that complexity-entropy values of $A L$ overlap the fBm values for all sub-sampling parameters $\tau$. As we increase $\tau$, the entropy for $A L$ increases, and the time-series becomes indistinguishable from fractional Brownian motion with antipersistent increments, i.e., with Hurst exponents less than 0.5.

It is natural to ask if the observed characterisation of $A L$ structures ranging between a few minutes to several hours is shared by other auroral current indices. In Figures 5 and 6, we show the dependence of the Jensen-Shannon complexity for all 12 months of 2010 for $A E$ and $A U$ respectively. The range of parameters $d, \tau$ and the legend are the same as in Figure 3. Once again the complexity value is relatively small for both indices. It peaks at low $\tau$ and decays for large $\tau$ values. Similarly to $A L$, we note that $A E$ also experiences a local maximum in complexity values for embedded delay ranging between $\tau \simeq 10$ and $\tau \simeq 50$, albeit more pronounced and for different months (with the most 
obvious month being April). The complexity for $A U$ on the other hand experiences local maximum for embedded delay ranging between $\tau \simeq 30$ and $\tau \simeq 200$ minutes, with the notable exception of June, July and November where the complexity monotonically decreases until $\tau \simeq 200$ and plateau at very low values thereafter. In Figure 7 we have reproduced the complexity-entropy plane for $A L$ (blue stars) and fBm (red dots) and complemented it with values for $A E$ (black circles) and $A U$ (magenta lozenges) across the month of August 2010. Similarly as for $A L, A U$ and $A E$ are highly stochastic across timescales ranging between a few minutes to 10 hours. We note that the same conclusion are equally valid for any other set of months (not shown).

\section{Discussion and Conclusion}

Using the permutation entropy developed by Bandt and Pompe (2002) and the complexityentropy plane methodology developed by Rosso et al. (2007) we have demonstrated that geomagnetic indices have larger complexity (structures) and lower entropy (uncertainty) on small timescales of $\tau<10$ than on timescales of $\tau>10$ minutes. Nonetheless, auroral geomagnetic indices are indistinguishable from stochastic processes, overlapping with fractional Brownian processes on timescales ranging between a few minutes to 10 hours. Our results are therefore inconsistent with earlier studies of (Baker et al., 1990; Pavlos et al., 1992; D. Roberts et al., 1991; D. A. Roberts, 1991; Vassiliadis et al., 1991; Vassiliadis et al., 1990) indicating that low-dimensional dynamical systems with chaotic properties might arise in geomagnetic current patterns.

In a similar study, Consolini and De Michelis (2014) also use permutation entropy as a measure of complexity to study the statistical properties of SYM-H timeseries spanning the period of January 2000 to December 2004. In their study, Consolini and De Michelis (2014) showed that permutation entropy computed on moving time windows was capable of capturing the rapid and local dynamical changes of $S Y M-H$. During storms, intermittency and the non-stationary nature of the fluctuations of $S Y M-H$ was shown to correlate with lower permutation entropy and higher complexity during quiet times. This result is consistent with Figures 4 and 7 showing that for lower $\tau$ values, structures in auroral currents have higher complexity and lower entropy.

Following the work of Balasis et al. (2006); Consolini, De Marco, and De Michelis (2013) and Osmane et al. (2015), our study also provides additional means to characterise large- 
scale and small-scale fluctuations originating in different physical processes. In a statistical study covering 17 years of OMNI data, (Osmane et al., 2015) showed that probability distribution functions of AL responded in a nontrivial yet coherent fashion to various solar wind properties and ULF fluctuation amplitudes. For strongly southward IMF, the AL distribution was characterised by a decrease of the skewness, a shift of the peak from $-30 \mathrm{nT}$ to $-200 \mathrm{nT}$ and a broadening of the distribution core. During northward IMF, the distribution in AL was instead characterised by a large reduction in the standard deviation and weight in the tail. Despite the different responses of the distribution function of AL for northward and southward IMF, the non-Gaussian changes were all occurring on timescales ranging between 10 and 40 minutes, similarly to the larger complexity structures observed in $A L$ and $A E$ on comparable timescales(i.e. comparable $\tau$ values), and associated with intermittent fluctuations. In Osmane et al. (2015), the authors argued that the non-Gaussian properties in the PDF of $A L$ occurring on timescales of the order of $\tau \sim 10-20$ minutes could be driven in part by viscous processes (Axford \& Hines, 1961), such as Kelvin-Helmholtz instability (Nykyri \& Otto, 2001) and kinetic Alfven waves (Johnson \& Cheng, 1997, 2001).

Whereas the mapping of auroral indices into the complexity-entropy plane was done independently of solar wind properties, follow-up studies could combine the methodology described in (Osmane et al., 2015), and the one presented here to distinguish coherent geomagnetic responses to upstream solar wind conditions from internal magnetospheric dynamic processes. Future studies will be extended to other geomagnetic indices and focus particularly on different solar wind driving conditions that might explain the enhancement in complexity on small timescales of $\tau<40$ minutes. Of particular interest, delineating storms in terms of solar wind conditions and statistics might indicate the contribution of magnetospheric dynamics in the triggering of storm activity and the nature of the nonlinear driving on timescales of minutes where viscous processes take place (Axford \& Hines, 1961; Chaston et al., 2007; Freeman, Warren, \& Maguire, 1968; Hasegawa et al., 2004, 2006; Johnson \& Cheng, 1997, 2001; Lee, Johnson, \& Ma, 1994; Nykyri \& Otto, 2001; Nykyri et al., 2006), to hours where geomagnetic storms unfold (T. Pulkkinen, 2007).

Finally, it should be kept in mind that auroral geomagnetic indices do not necessarily account for the detailed spatial variations of the magnetic field. For instance, as the auroral electrojet expands in large storms, high latitude observatories at which AE is de- 
rived experience lower magnetic field variations. Additionally, auroral geomagnetic indices cannot capture the complexity of the wider magnetospheric system since they are constructed as a multi-dimensional mapping of several observatories and reduced to a single proxy parameter. In the case of $\mathrm{AL}$, it serves as a proxy for the energy transmitted into the ionosphere. We can therefore not exclude the possibility that spatial and/or temporal variations associated with various magnetospheric processes could be modelled in terms of a deterministic set of equations, albeit one that is not as low-dimensional as previous authors suggested. Rather, our analysis provides an answer to a much narrower question: Can we model fluctuations in auroral geomagnetic indices as low-dimensional chaotic attractors, and consequently reduce a system a priori composed of a very large number degrees of freedom to one with a few degrees of freedom? Though our answer is undoubtedly in the negative, our analysis does not preclude the existence of a highdimensional chaotic systems or one based on self-organised critical models (Sharma et al, 2016).

\section{Acknowledgments}

This work was supported by Academy of Finland grants \#267073/2013 and \#297688/2015. AO would also like to thank James Weygand and Joe Borovsky for useful discussions on the use of the complexity-entropy approach to space data. The OMNI data used in this paper is available at http://omniweb.gsfc.nasa.gov free of charge

\section{References}

Axford, W. I., \& Hines, C. O. (1961). A unifying theory of high-latitude geophysical phenomena and geomagnetic storms. Can. J. Phys., 39, 1433 . doi: 10.1139/ p61-172

Baker, D., Klimas, A., McPherron, R., \& Büchner, J. (1990). The evolution from weak to strong geomagnetic activity: An interpretation in terms of deterministic chaos. Geophysical research letters, 17(1), 41-44.

Balasis, G., Daglis, I. A., Kapiris, P., Mandea, M., Vassiliadis, D., \& Eftaxias, K. (2006, December). From pre-storm activity to magnetic storms: a transition described in terms of fractal dynamics. Annales Geophysicae, 24, 3557-3567. doi: 10.5194/angeo-24-3557-2006

Bandt, C., \& Pompe, B. (2002, April). Permutation Entropy: A Natural Complex- 
ity Measure for Time Series. Physical Review Letters, 88(17), 174102. doi: 10 .1103/PhysRevLett.88.174102

Berthelier, A., \& Menvielle, M. (1993). Geomagnetic data 1987, iaga indices: aa, am, kp, dst, ae, rapid variations. IAGA Bulletin, 32.

Chang, T. (1992). Low-dimensional behavior and symmetry breaking of stochastic systems near criticality-can these effects be observed in space and in the laboratory? Plasma Science, IEEE Transactions on, 20(6), 691-694.

Chaston, C., Wilber, M., Mozer, F., Fujimoto, M., Goldstein, M., Acuna, M., ... Fazakerley, A. (2007). Mode conversion and anomalous transport in kelvinhelmholtz vortices and kinetic alfvén waves at the earth?s magnetopause. Physical review letters, 99(17), 175004.

Consolini, G., De Marco, R., \& De Michelis, P. (2013). Intermittency and multifractional brownian character of geomagnetic time series. Nonlinear Processes in Geophysics, 20(4), 455-466.

Consolini, G., \& De Michelis, P. (2014). Permutation entropy analysis of complex magnetospheric dynamics. Journal of Atmospheric and Solar-Terrestrial Physics, 115, 25-31.

Consolini, G., \& Marcucci, M. (1997). Multifractal structure and intermittence in the ae index time series. Nuovo cimento della Società italiana di fisica. C, 20(6), 939-949.

Dobias, P., \& Wanliss, J. A. (2009, May). Intermittency of storms and substorms: is it related to the critical behaviour? Annales Geophysicae, 27, 2011-2018. doi: 10.5194/angeo-27-2011-2009

Farris, M. H., \& Russell, C. T. (1994, September). Determining the standoff distance of the bow shock: Mach number dependence and use of models. J. Geophys. Res. Space Phys., 99, 17681. doi: 10.1029/94JA01020

Freeman, J., Warren, C., \& Maguire, J. (1968). Plasma flow directions at the magnetopause on january 13 and 14, $1967 . \quad J o u r n a l$ of Geophysical Research, 73(17), 5719-5731.

Gollub, J. P., \& Swinney, H. L. (1975). Onset of turbulence in a rotating fluid. Physical Review Letters, 35(14), 927.

Grassberger, P., \& Procaccia, I. (1983). Characterization of strange attractors. Physical review letters, 50(5), 346. 
Hasegawa, H., Fujimoto, M., Phan, T.-D., Reme, H., Balogh, A., Dunlop, M., ... TanDokoro, R. (2004). Transport of solar wind into earth's magnetosphere through rolled-up kelvin-helmholtz vortices. Nature, 430(7001), 755-758.

Hasegawa, H., Fujimoto, M., Takagi, K., Saito, Y., Mukai, T., \& Rème, H. (2006). Single-spacecraft detection of rolled-up kelvin-helmholtz vortices at the flank magnetopause. Journal of Geophysical Research: Space Physics (1978-2012), 111(A9).

Johnson, J. R., \& Cheng, C. Z. (1997). Kinetic Alfvén waves and plasma transport at the magnetopause. Geophys. Res. Lett., 24, 1423-1426. doi: 10.1029/ 97GL01333

Johnson, J. R., \& Cheng, C. Z. (2001). Stochastic ion heating at the magnetopause due to kinetic Alfvén waves. Geophys. Res. Lett., 28, 4421-4424. doi: 10.1029/ 2001GL013509

King, J. H., \& Papitashvili, N. E. (2005, February). Solar wind spatial scales in and comparisons of hourly Wind and ACE plasma and magnetic field data. J. Geophys. Res. Space Phys., 110, 2104. doi: 10.1029/2004JA010649

Klimas, A. J., Valdivia, J., Vassiliadis, D., Baker, D. N., Hesse, M., \& Takalo, J. (2000, August). Self-organised criticality in the substorm phenomenon and its relation to localised reconnection in the magnetospheric plasma sheet. $J$. Geophys. Res. Space Phys., 105, 18765-18780. doi: 10.1029/1999JA000319

Klimas, A. J., Vassiliadis, D., Baker, D. N., \& Roberts, D. A. $\quad$ (1996, June). The organized nonlinear dynamics of the magnetosphere. J. Geophys. Res. Space Phys., 101, 13089-13114. doi: 10.1029/96JA00563

Landau, L. D. (1944). On the problem of turbulence. In Dokl. akad. nauk sssr (Vol. 44, pp. 339-349).

Lee, L., Johnson, J. R., \& Ma, Z. (1994). Kinetic alfvén waves as a source of plasma transport at the dayside magnetopause. Journal of Geophysical Research: Space Physics (1978-2012), 99(A9), 17405-17411.

Lovejoy, S., \& Schertzer, D. (1998). Stochastic chaos and multifractal geophysics. Chaos, Fractals and models, 96, 38-52.

Maggs, J., \& Morales, G. (2013). Permutation entropy analysis of temperature fluctuations from a basic electron heat transport experiment. Plasma Physics and Controlled Fusion, 55(8), 085015. 
Mandelbrot, B. B., \& Van Ness, J. W. (1968). Fractional brownian motions, fractional noises and applications. SIAM review, 10(4), 422-437.

Newell, P., Sotirelis, T., Liou, K., Meng, C.-I., \& Rich, F. (2007). A nearly universal solar wind-magnetosphere coupling function inferred from 10 magnetospheric state variables. Journal of Geophysical Research: Space Physics (1978-2012), 112(A1)

Nykyri, K., \& Otto, A. (2001). Plasma transport at the magnetospheric boundary due to reconnection in kelvin-helmholtz vortices. Geophys. Res. Lett., 28(18), $3565-3568$.

Nykyri, K., Otto, A., Lavraud, B., Mouikis, C., Kistler, L. M., Balogh, A., \& Rème, H. (2006, October). Cluster observations of reconnection due to the KelvinHelmholtz instability at the dawnside magnetospheric flank. Ann. Geophys., 24, 2619-2643. doi: 10.5194/angeo-24-2619-2006

Osborne, A. R., \& Provenzale, A. (1989). Finite correlation dimension for stochastic systems with power-law spectra. Physica D: Nonlinear Phenomena, 35(3), $357-381$.

Osmane, A., Dimmock, A., Naderpour, R., Pulkkinen, T., \& Nykyri, N. (2015). The impact of solar wind ulf $b_{z}$ fluctuations on geomagnetic activity for viscous timescales during strongly northward and southward imf. J. Geophys. Res., 2169-9402. doi: 10.1002/2015JA021505

Ott, E. (2002). Chaos in dynamical systems. Cambridge university press.

Pavlos, G., Kyriakou, G., Rigas, A., Liatsis, P., Trochoutsos, P., \& Tsonis, A.

(1992). Evidence for strange attractor structures in space plasmas. In Annales geophysicae (Vol. 10, pp. 309-322).

Prichard, D., \& Price, C. (1992). Spurious dimension estimates from time series of geomagnetic indices. Geophysical research letters, 19(15), 1623-1626.

Pulkkinen, A., Klimas, A., Vassiliadis, D., \& Uritsky, V. (2006). Role of stochastic fluctuations in the magnetosphere-ionosphere system: A stochastic model for the ae index variations. Journal of Geophysical Research: Space Physics (1978-2012), 111(A10).

Pulkkinen, T. (2007). Space weather: terrestrial perspective. Living Rev. Solar Phys, 4(1).

Riedl, M., Muller, A., \& Wessel, N. (2013). Practical considerations of permuta- 
tion entropy. Eur. Phys. J. Special Topics, 222, 249. doi: 10.1140/epjst/e2013 $-01862$

Roberts, D., Baker, D., Klimas, A., \& Bargatze, L. (1991). Indications of low dimensionality in magnetospheric dynamics. Geophysical Research Letters, 18(2), $151-154$.

Roberts, D. A. (1991). Is there a strange attractor in the magnetosphere? Journal of Geophysical Research: Space Physics (1978-2012), 96(A9), 16031-16046.

Rosso, O. A., Larrondo, H. A., Martin, M. T., Plastino, A., \& Fuentes, M. A. (2007, October). Distinguishing Noise from Chaos. Physical Review Letters, 99(15), 154102. doi: 10.1103/PhysRevLett.99.154102

Rosso, O. A., Olivares, F., Zunino, L., De Micco, L., Aquino, A. L. L., Plastino, A., \& Larrondo, H. A. (2013, April). Characterization of chaotic maps using the permutation Bandt-Pompe probability distribution. European Physical Journal $B, 86$, 116. doi: $10.1140 /$ epjb/e2013-30764-5

Ruelle, D., \& Takens, F. (1971). On the nature of turbulence. Commun. math. phys, $20(3), 167-192$.

Shan, L.-H., Hansen, P., Goertz, C., \& Smith, R. (1991). Chaotic appearance of the ae index. Geophysical Research Letters, 18(2), 147-150.

Sharma et al, A. S. (2016). 25 years of self-organized criticality: space and laboratory plasmas. Space Sci. Rev., 198, 167-216. doi: 10.1007/s11214-015-0225-0

Tanskanen, E., Pulkkinen, T., Koskinen, H., \& Slavin, J. (2002). Substorm energy budget during low and high solar activity: 1997 and 1999 compared. Journal of Geophysical Research: Space Physics (1978-2012), 107(A6), SMP-15.

Uritsky, V., Pudovkin, M., \& Steen, A. (2001). Geomagnetic substorms as perturbed self-organized critical dynamics of the magnetosphere. Journal of Atmospheric and Solar-Terrestrial Physics, 63(13), 1415-1424.

Uritsky, V. M., Klimas, A. J., \& Vassiliadis, D. (2006). Analysis and prediction of high-latitude geomagnetic disturbances based on a self-organized criticality framework. Advances in Space Research, 37(3), 539-546.

Uritsky, V. M., \& Pudovkin, M. I. （1998, December). Low frequency 1/f-like fluctuations of the AE-index as a possible manifestation of self-organized criticality in the magnetosphere. Annales Geophysicae, 16, 1580-1588. doi: $10.1007 / \mathrm{s} 00585-998-1580-\mathrm{x}$ 
Valdivia, J., Klimas, A., Vassiliadis, D., Uritsky, V., \& Takalo, J. ～(2003). Selforganization in a current sheet model. In Advances in space environment research (pp. 515-522). Springer.

Vassiliadis, D., Sharma, A., \& Papadopoulos, K. (1991). Lyapunov exponent of magnetospheric activity from al time series. Geophysical research letters, 18(8), 1643-1646.

Vassiliadis, D. V., Sharma, A. S., Eastman, T. E., \& Papadopoulos, K. (1990, October). Low-dimensional chaos in magnetospheric activity from AE time series. Geophysical research letters, 17, 1841-1844. doi: 10.1029/GL017i011p01841

Wanliss, J. A., Anh, V. V., Yu, Z.-G., \& Watson, S. (2005, August). Multifractal modeling of magnetic storms via symbolic dynamics analysis. Journal of Geophysical Research (Space Physics), 110, 8214. doi: 10.1029/2004JA010996

Wanliss, J. A., \& Dobias, P. (2007, April). Space storm as a phase transition. Journal of Atmospheric and Solar-Terrestrial Physics, 69, 675-684. doi: 10.1016/j .jastp.2007.01.001

Weck, P. J., Schaffner, D. A., Brown, M. R., \& Wicks, R. T. （2015, February). Permutation entropy and statistical complexity analysis of turbulence in laboratory plasmas and the solar wind. Physical Review E, 91(2), 023101. doi: 10.1103/PhysRevE.91.023101

Zanin, M., Zunino, L., Rosso, O. A., \& Papo, D. (2012, August). Permutation Entropy and Its Main Biomedical and Econophysics Applications: A Review. Entropy, 14, 1553-1577. doi: 10.3390/e14081553 

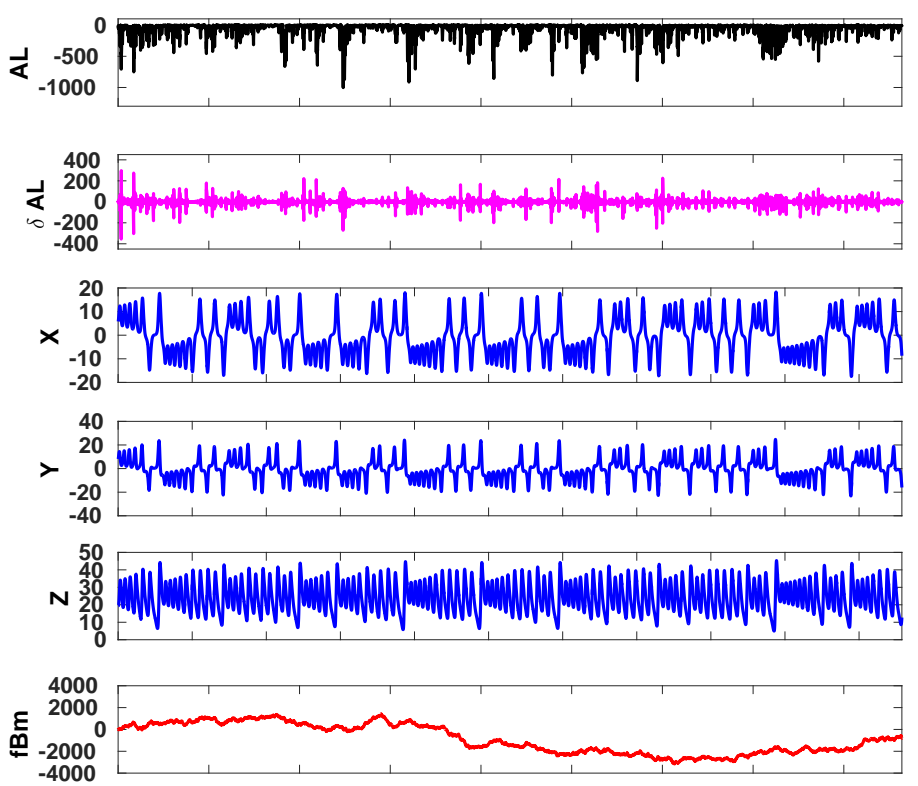

Figure 1. Top two panels show $\mathrm{AL}$ and $\delta A L=A L_{i+1}-A L_{i}$ time series for an interval of four months spanning 01-01-2009 to 30-04-2009. The panels with blue traces show time series for the Lorenz attractor with parameters $a=10, b=8 / 3, c=28$. The bottom panel shows an example of fractional Brownian motion with Hurst exponent $h_{u}=0.8$.

This article is protected by copyright. All rights reserved. 

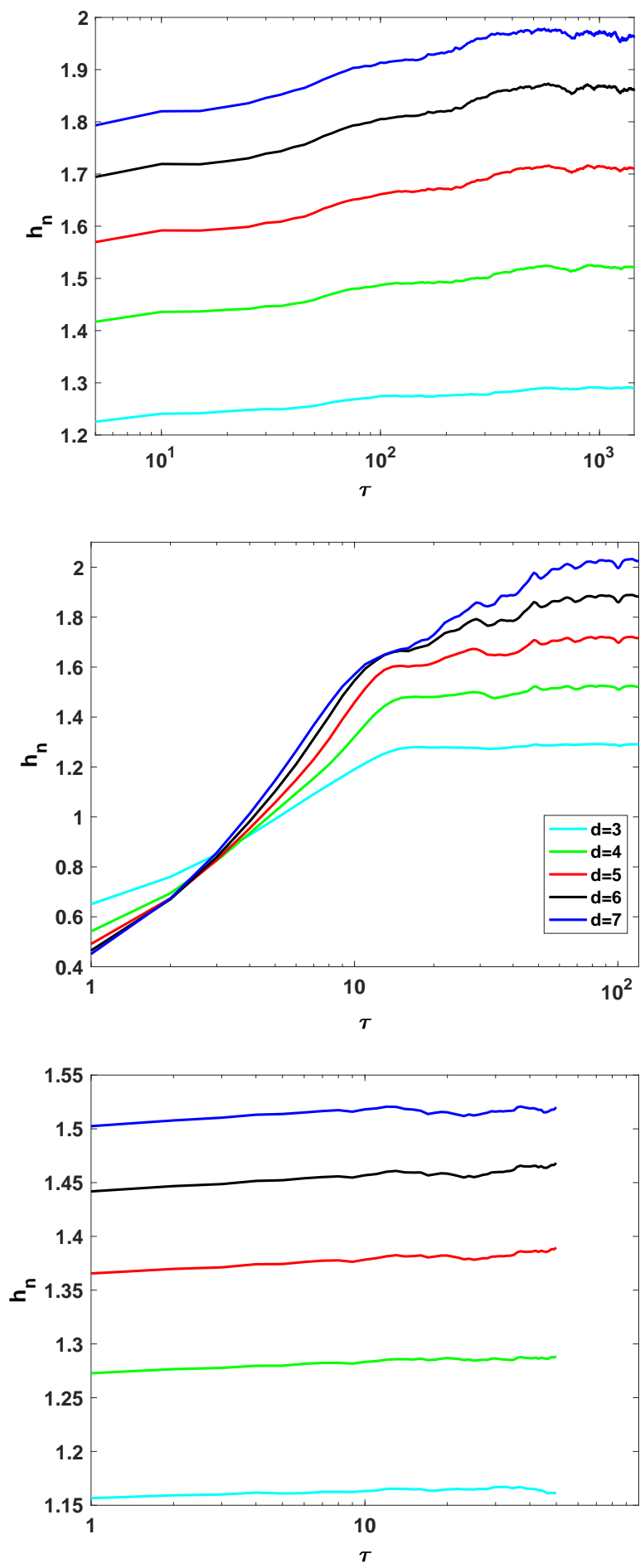

Figure 2. Permutation entropy as a function embedded delay $\tau$ and parametrised for embedding dimension $3 \leq d \leq 7$ for fractional Brownian motion with Hurst exponent $H=0.75$ (top panel), the Lorenz attractor (center panel) and AL (bottom panel). 

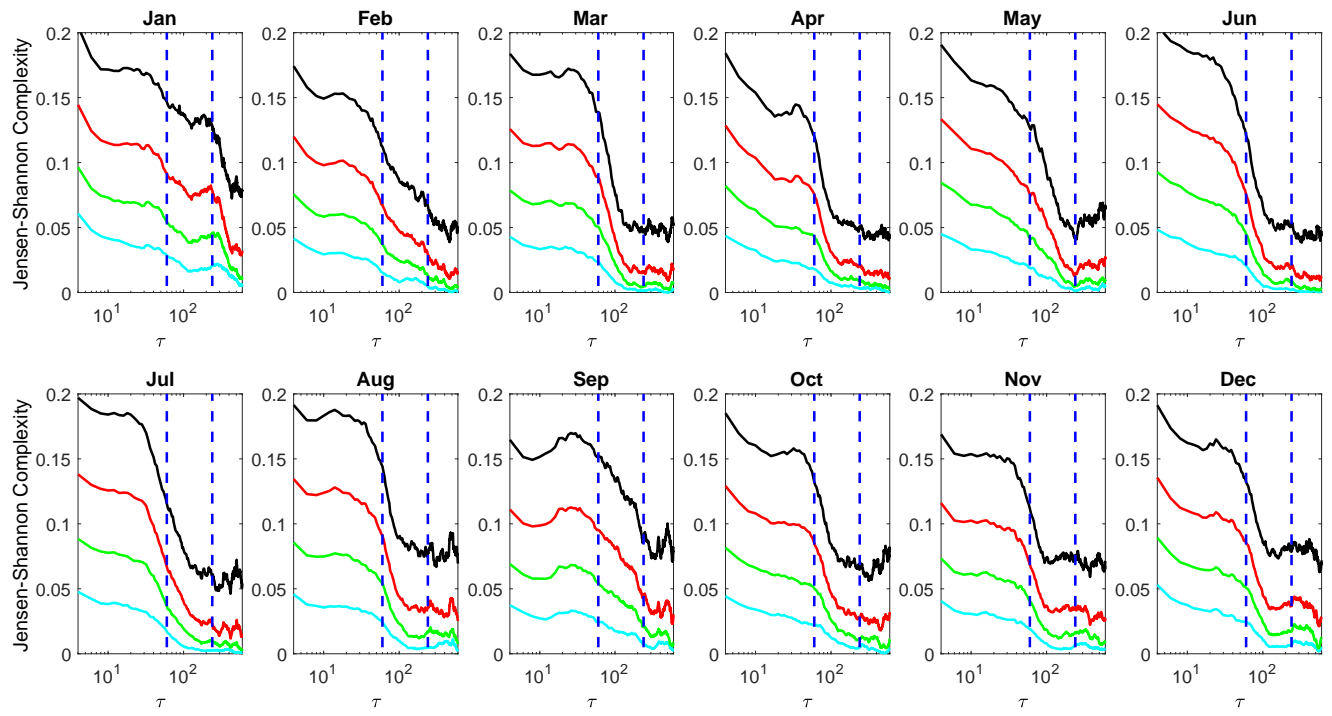

Figure 3. Jensen-Shannon Complexity versus embedded delay $\tau$ for all 12 months of 2010 of $A L$ values. The legend for the colour is the same as in Figure 2. The embedded delay $\tau$ has units of minutes and the dashed vertical line indicate the 60 minute and 240 minute marks.
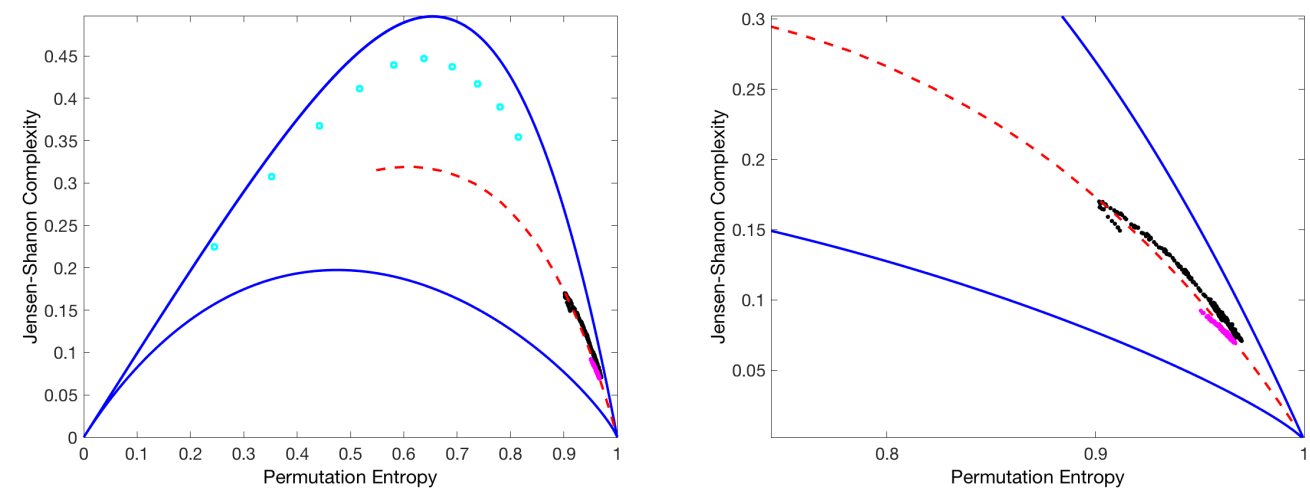

Figure 4. Complexity-entropy plane for $A L$ (black dots), $\delta A L$ (magenta dots), the Lorentz strange attractor (cyan squares), and fractional Brownian motion (red dash) on the left panel with a zoom in the low complexity high entropy part on the right panel. The blue curves represent the minimum and maximum entropy-complexity curve for an embedded parameter $d=6$. The permutation entropy and Jensen-Shannon complexity values for $A L$ and $\delta A L$ are computed for $4 \leq \tau \leq 600$ minutes. The complexity-values for AL reside along those for fractional-Brownian motion of Hurst exponents less than 0.5. 

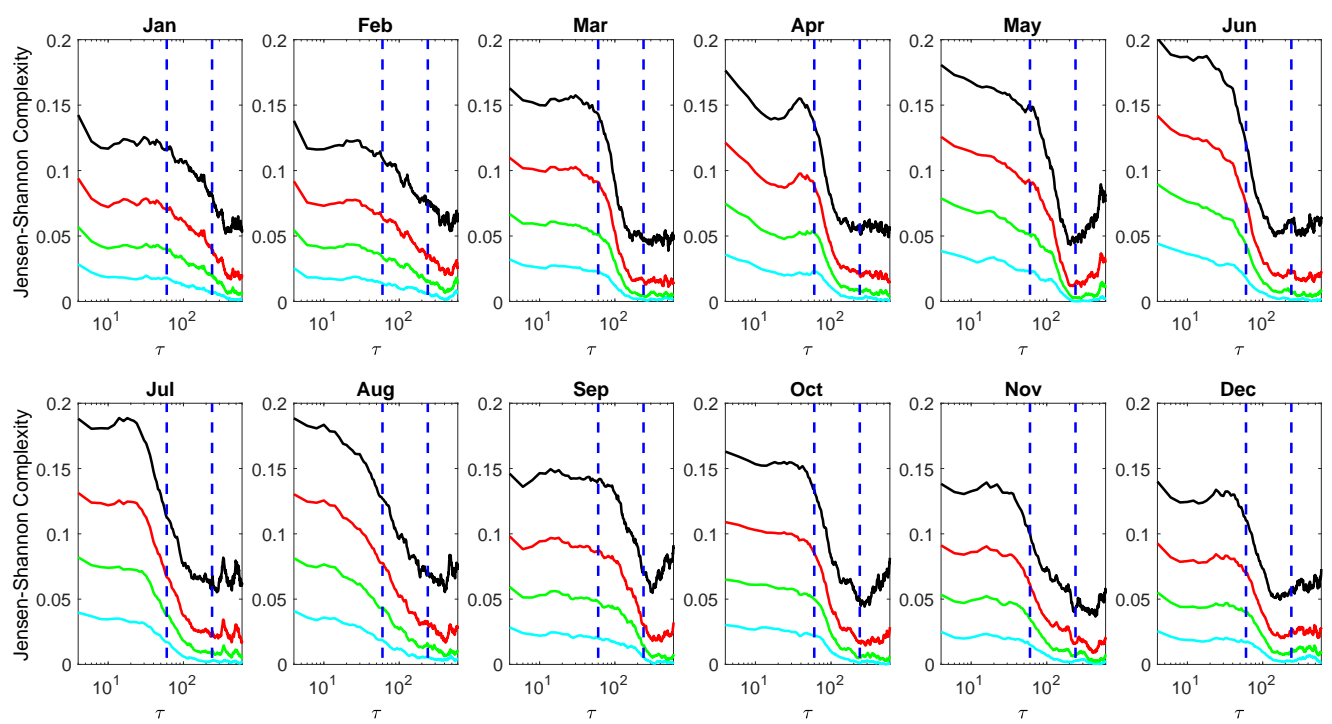

Figure 5. Jensen-Shannon Complexity versus embedded delay $\tau$ for all 12 months of 2010 of $A E$ values. The legend for the colour is the same as in Figure 2.
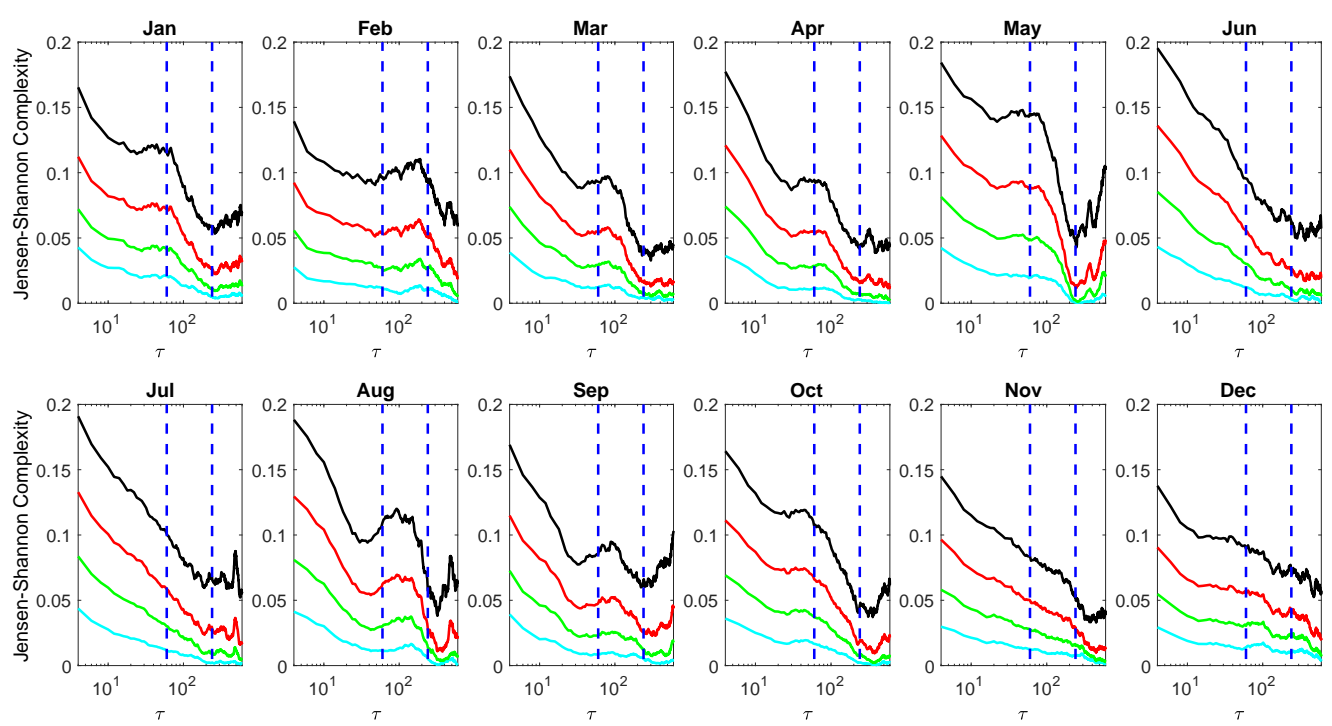

Figure 6. Jensen-Shannon Complexity versus embedded delay $\tau$ for all 12 months of 2010 of $A U$ values. The legend for the colour is the same as in Figure 2. 

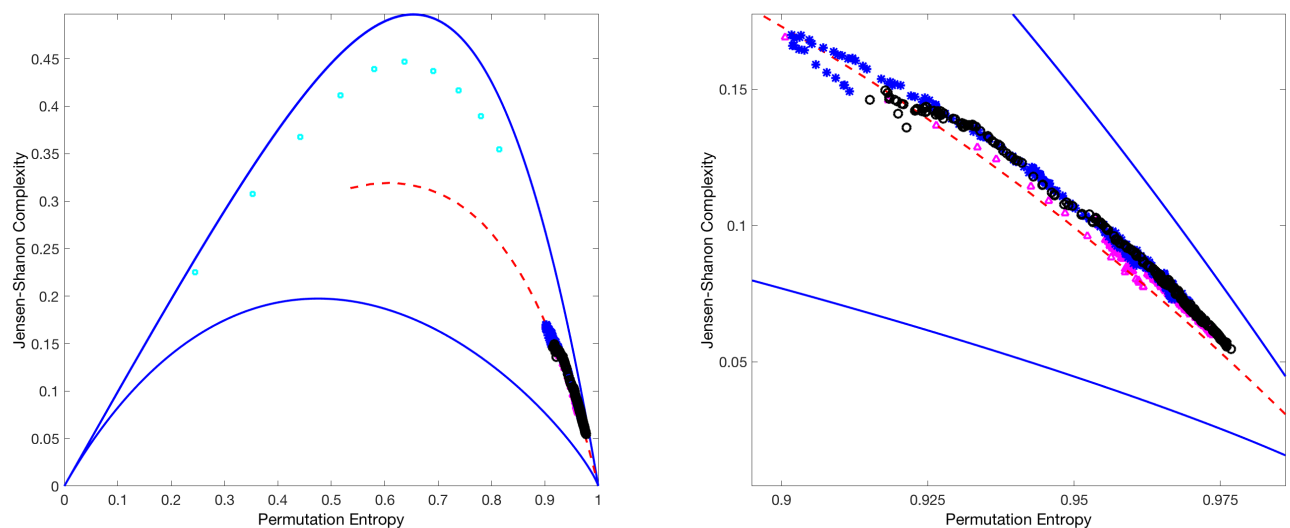

Figure 7. Complexity-Entropy plane for $A L$ (blue stars), $A U$ (magenta lozenge), $A E$ (black circles) and fractional Brownian motion (red dots) and $d=6$. The lowest entropy values are computed for $\tau=4$ minute, while the larger entropy values are for $\tau=600 \mathrm{~min}$. The complexityvalues for all three auroral geomagnetic indices reside along those for fractional-Brownian motion with Hurst exponents less than one half. 


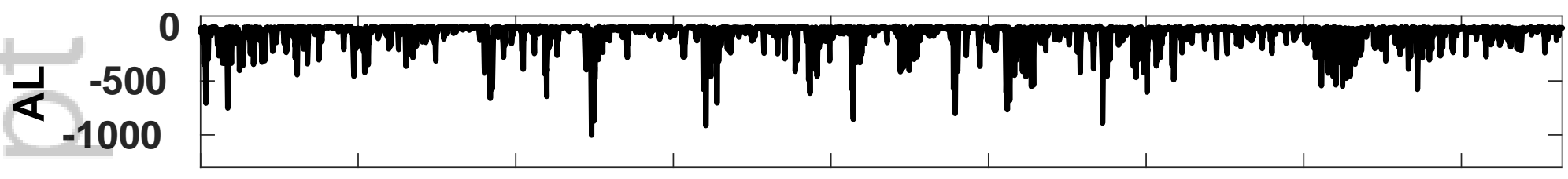

$\underset{-200}{200} \begin{gathered}400 \\ 20 \\ 0 \\ -200\end{gathered}$

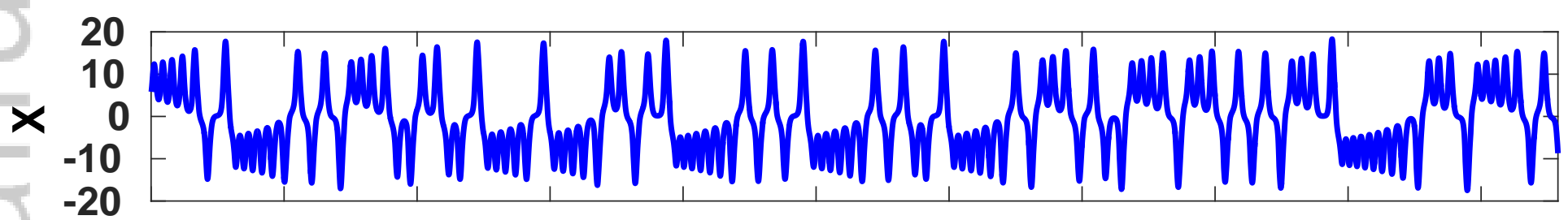

>
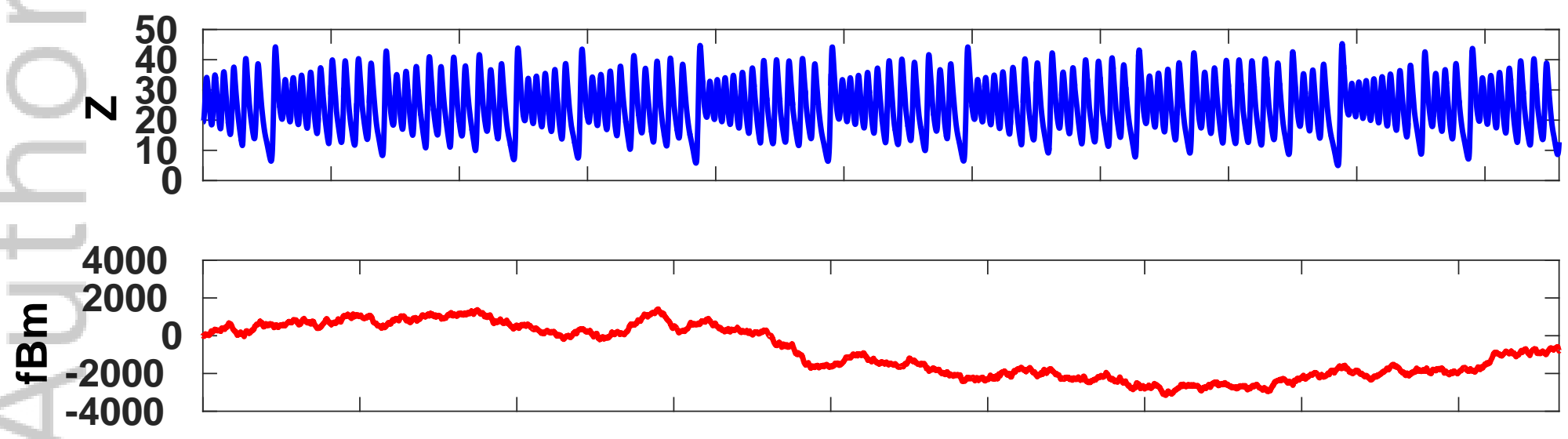

This article is protected by copyright. All rights reserved. 


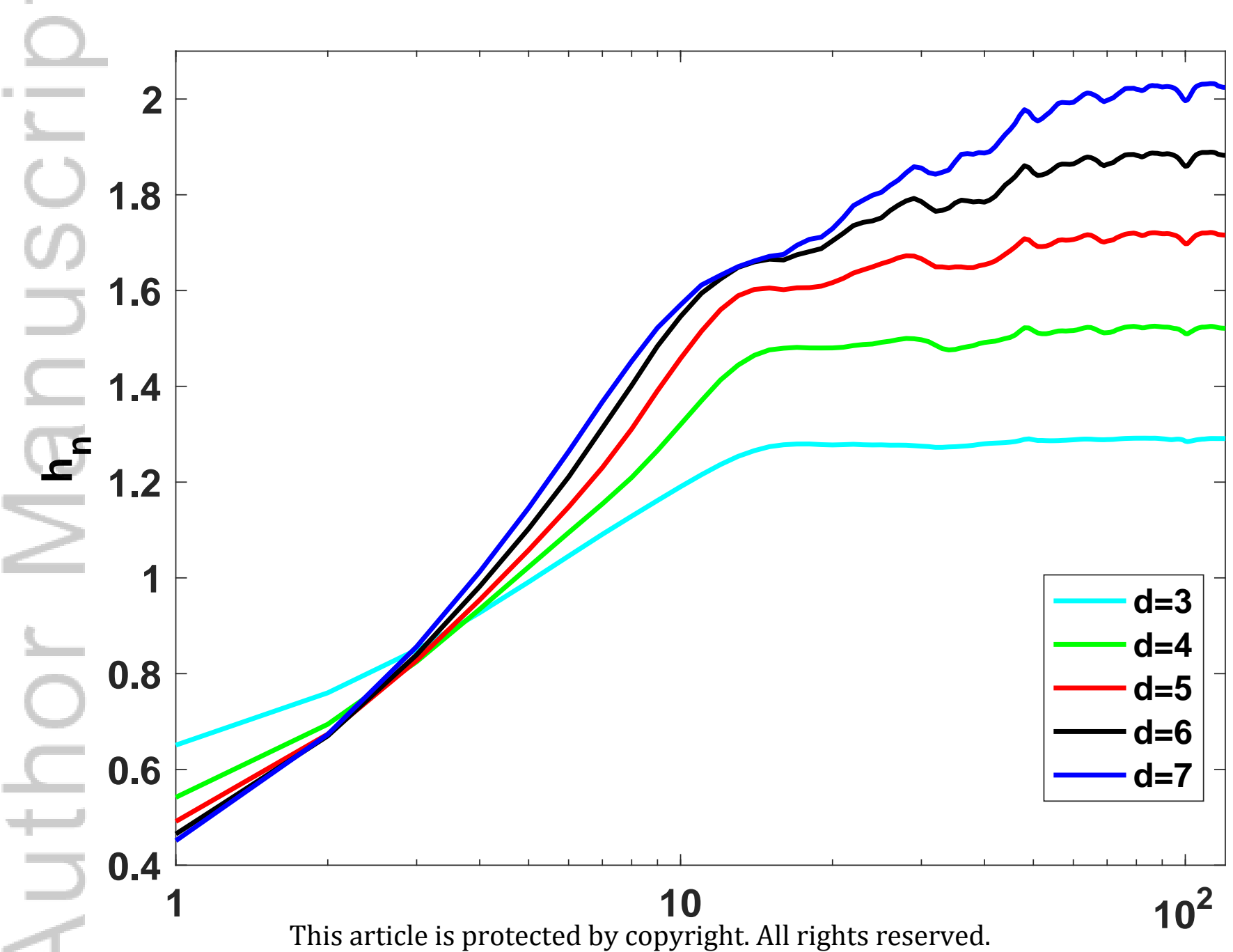

This article is protected by copyright. All rights reserved. 


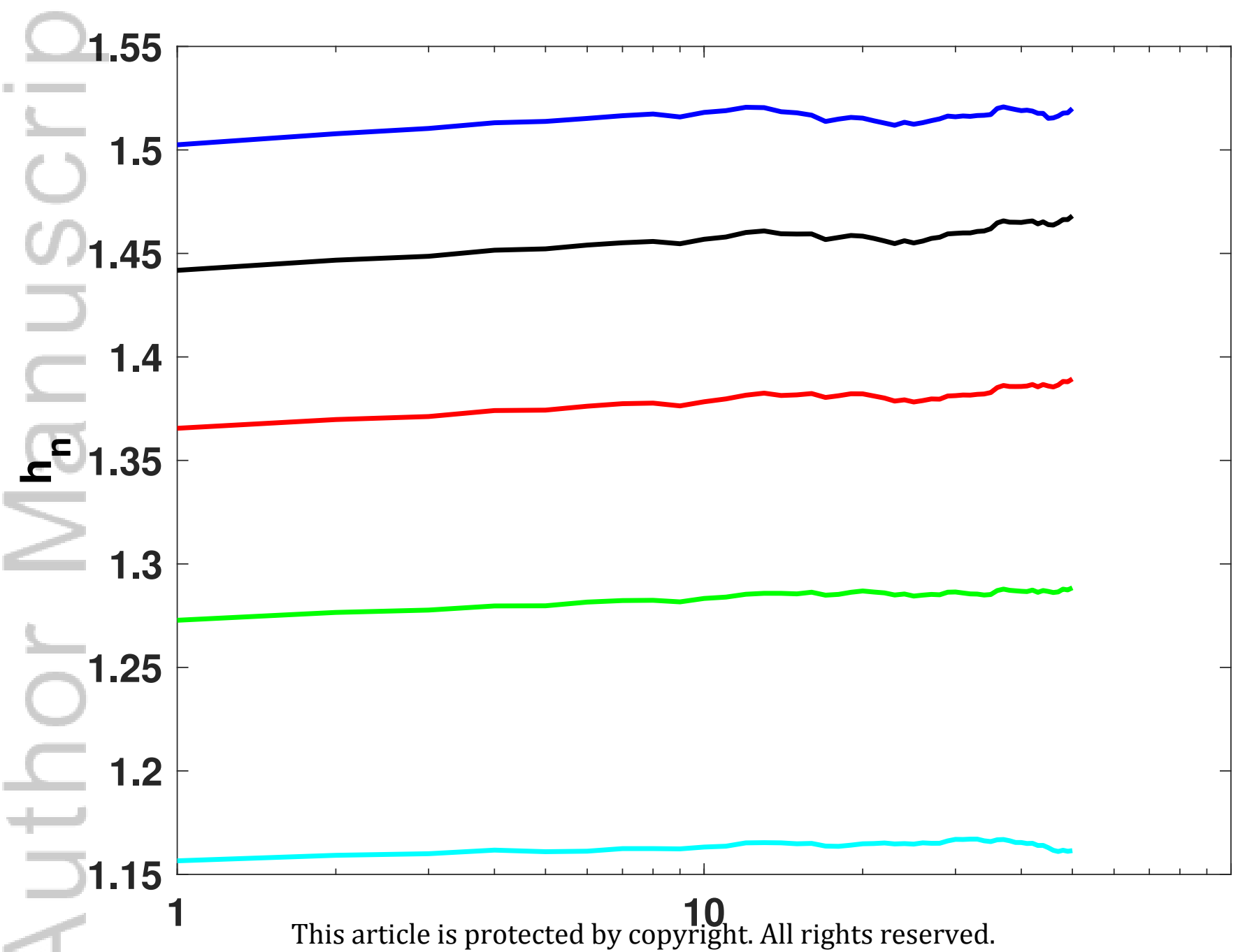



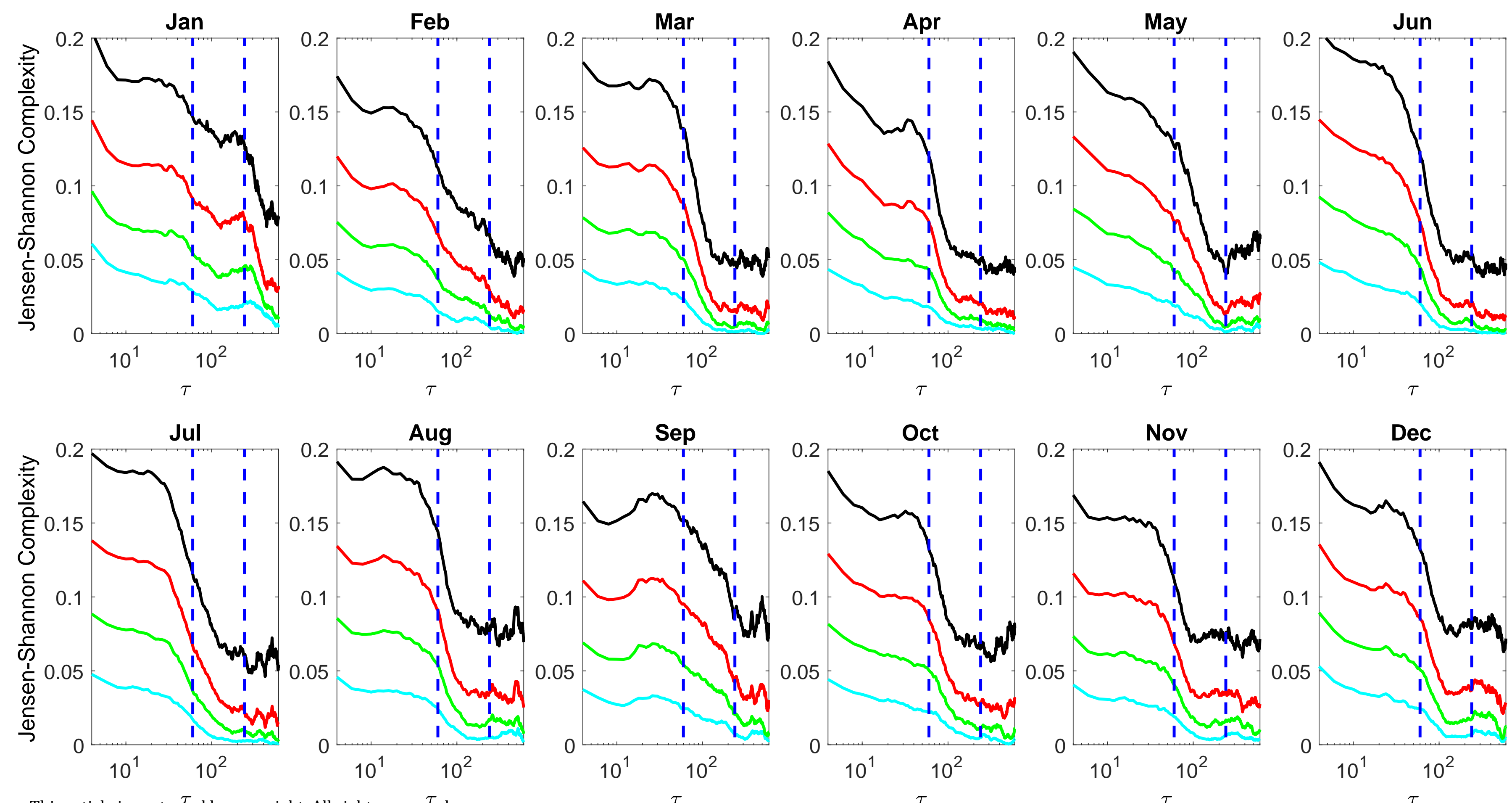

This article is protected by copyright. All rights reserfed. 


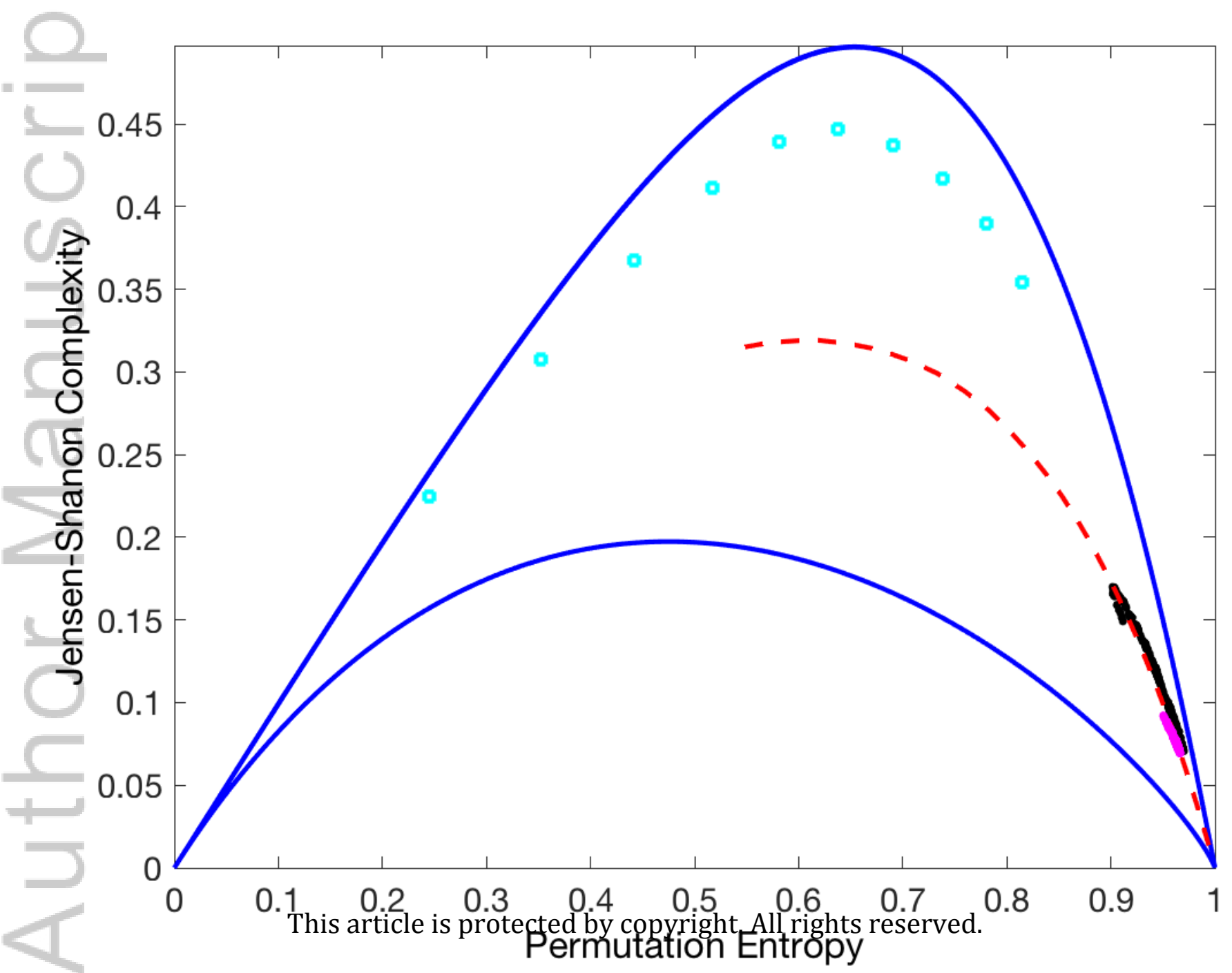


Figure 5.
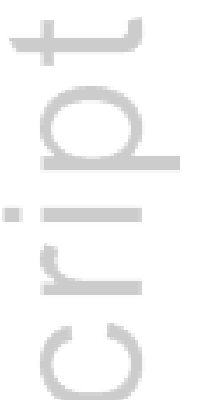

$\infty$
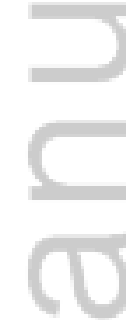

This article is protected by copyright. All rights reserved. 

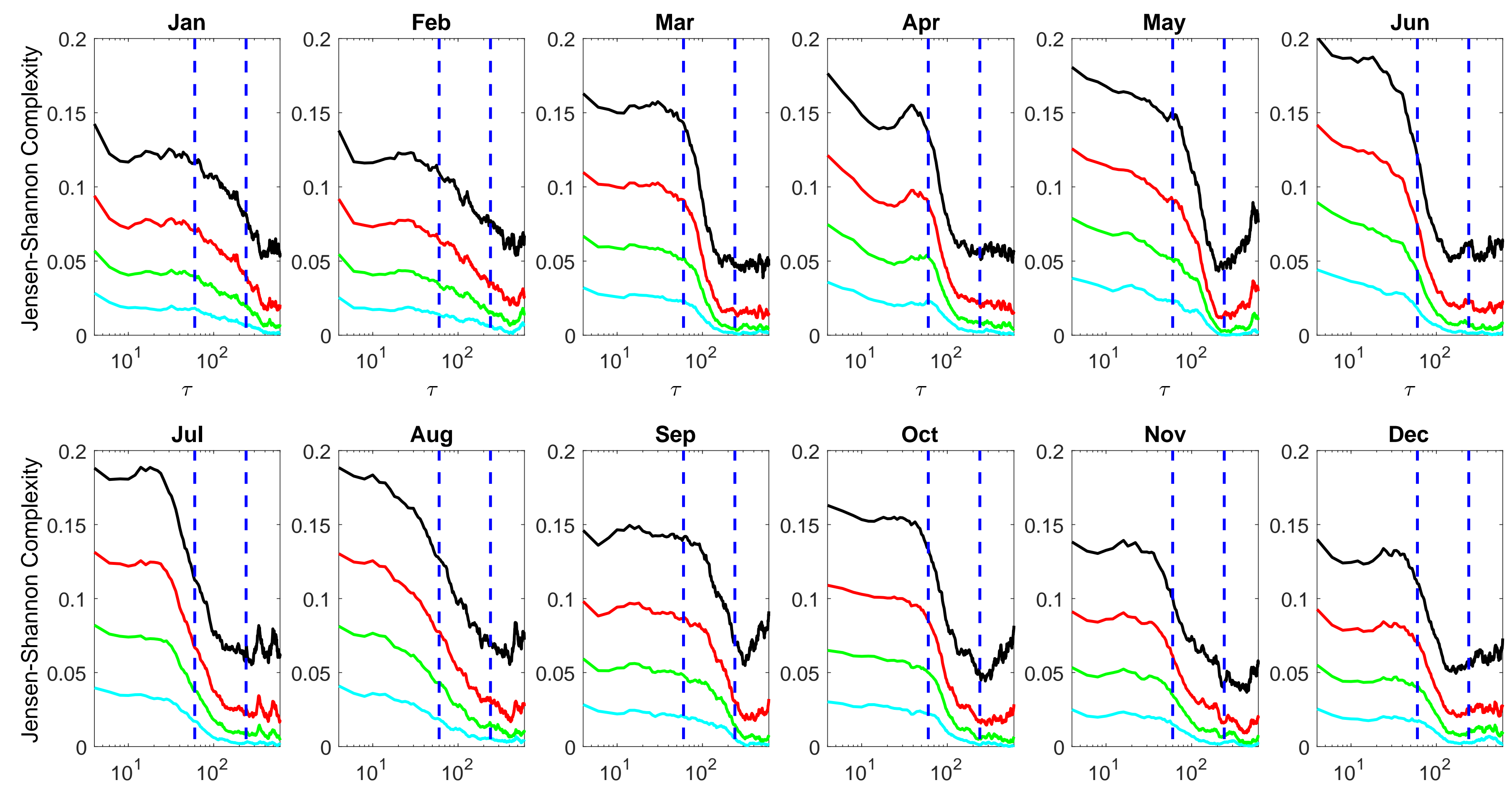

This article is protected by copyright. All rights reserved 

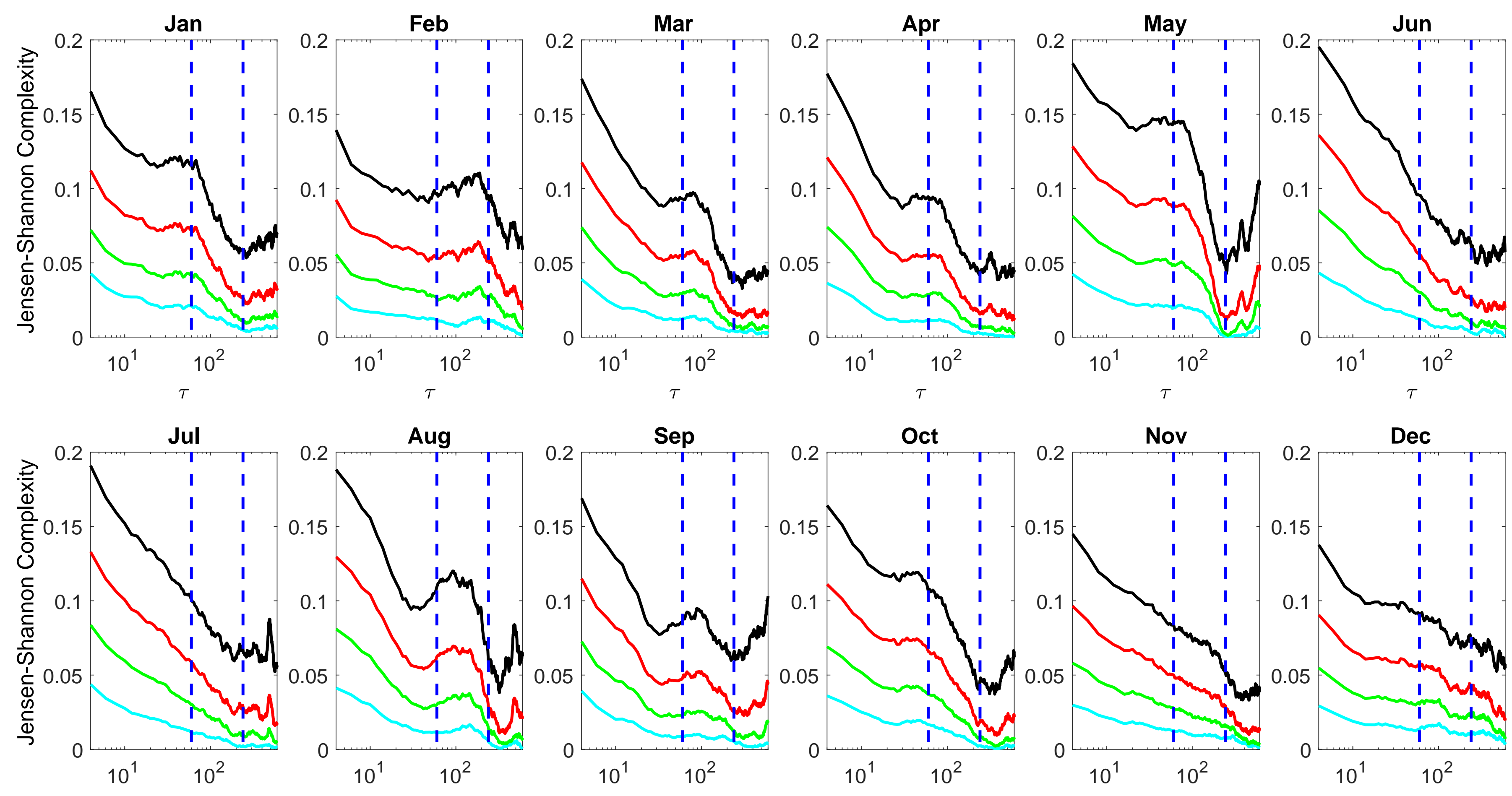

This article is protected by copyright. All rights reserved. 

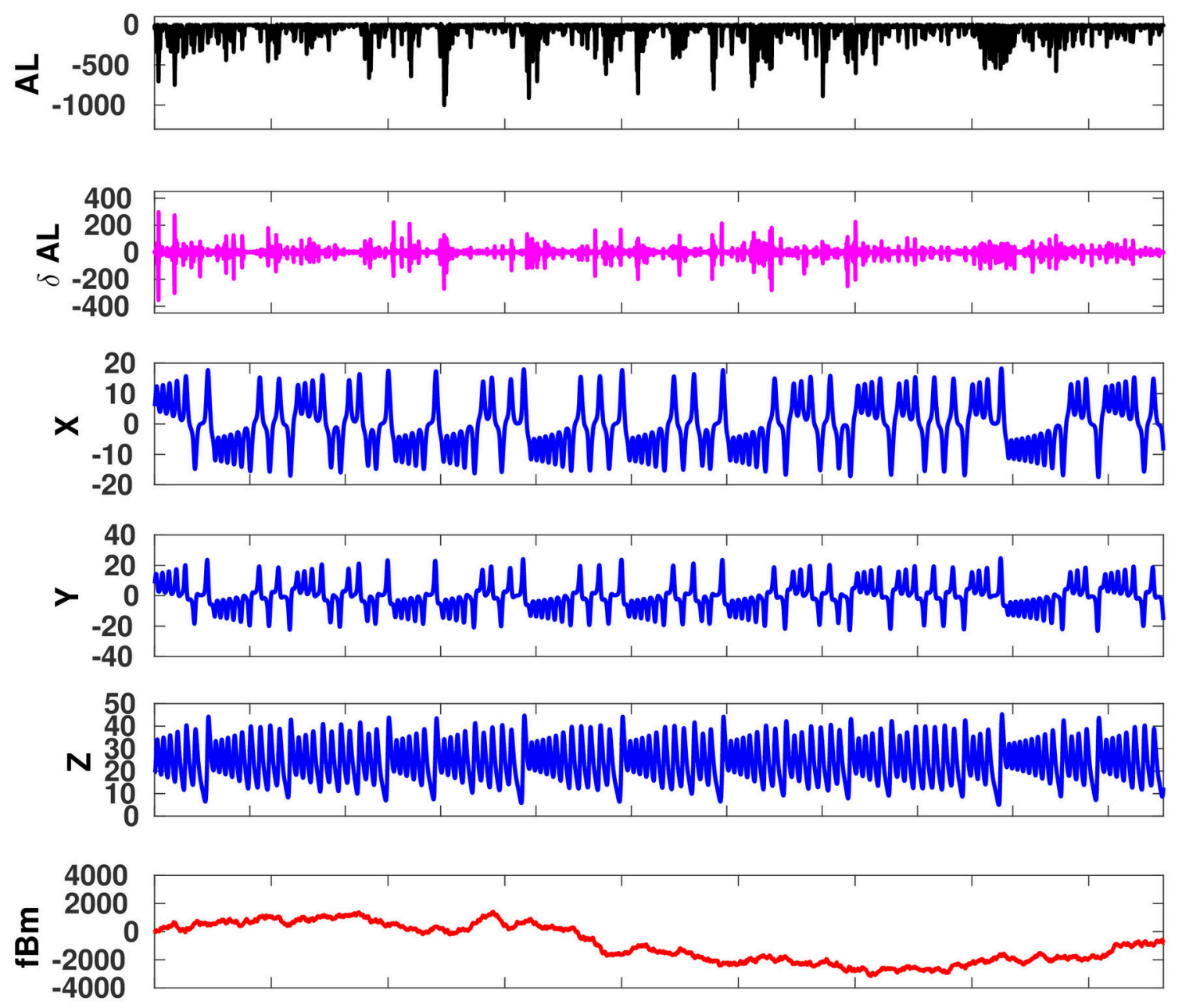

2018ja026248-t-f01-z-.eps

This article is protected by copyright. All rights reserved. 


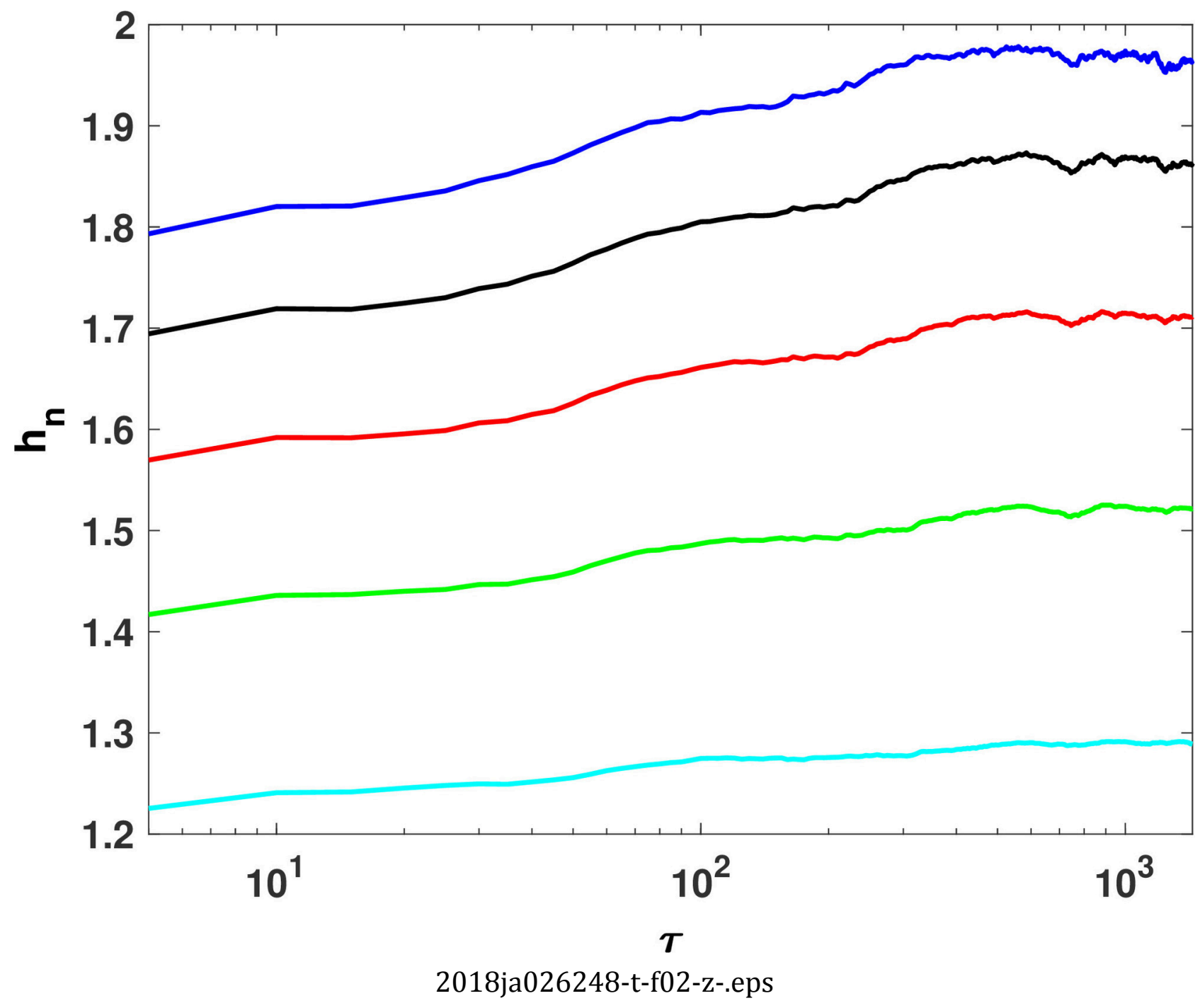

This article is protected by copyright. All rights reserved. 


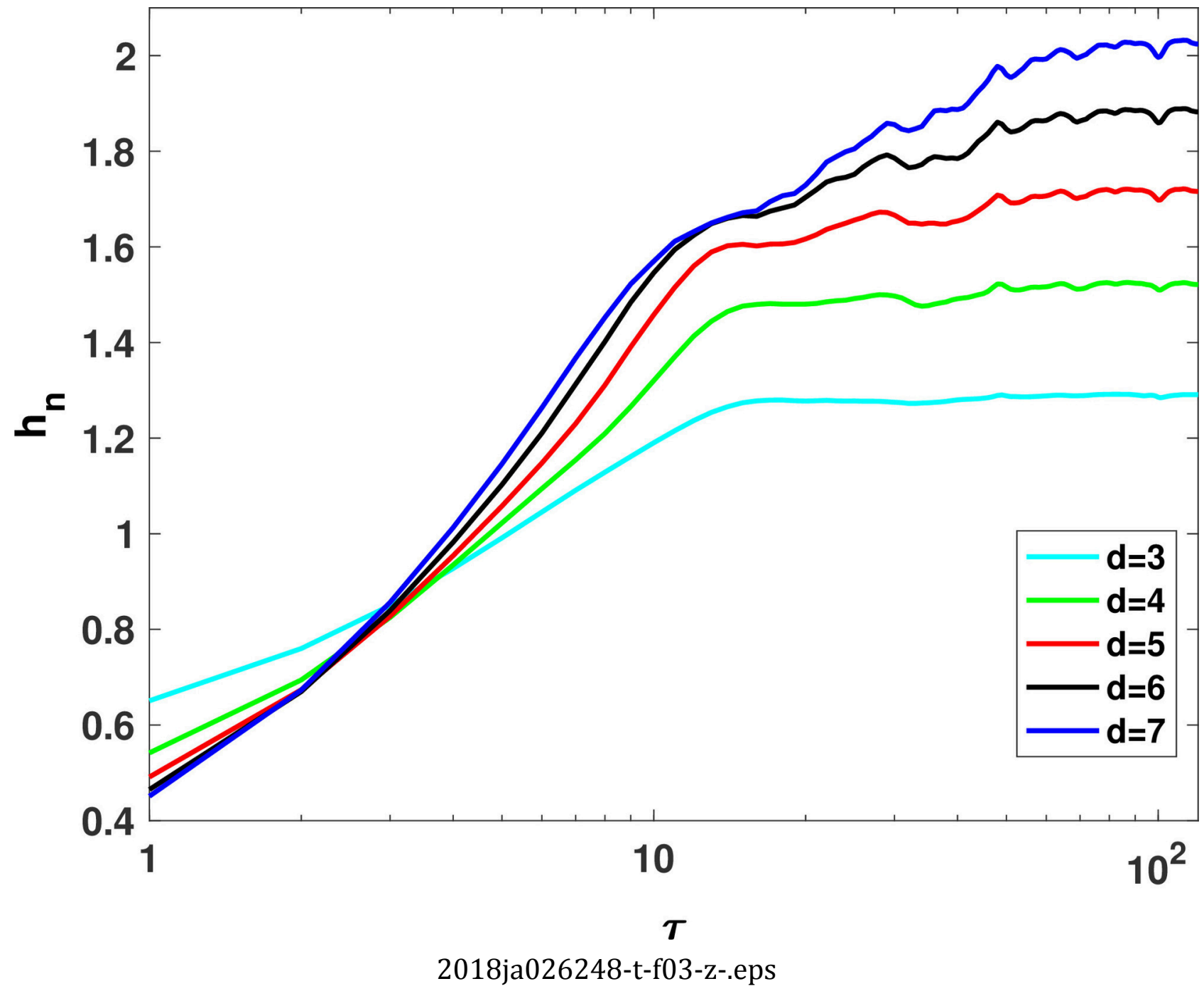

This article is protected by copyright. All rights reserved. 


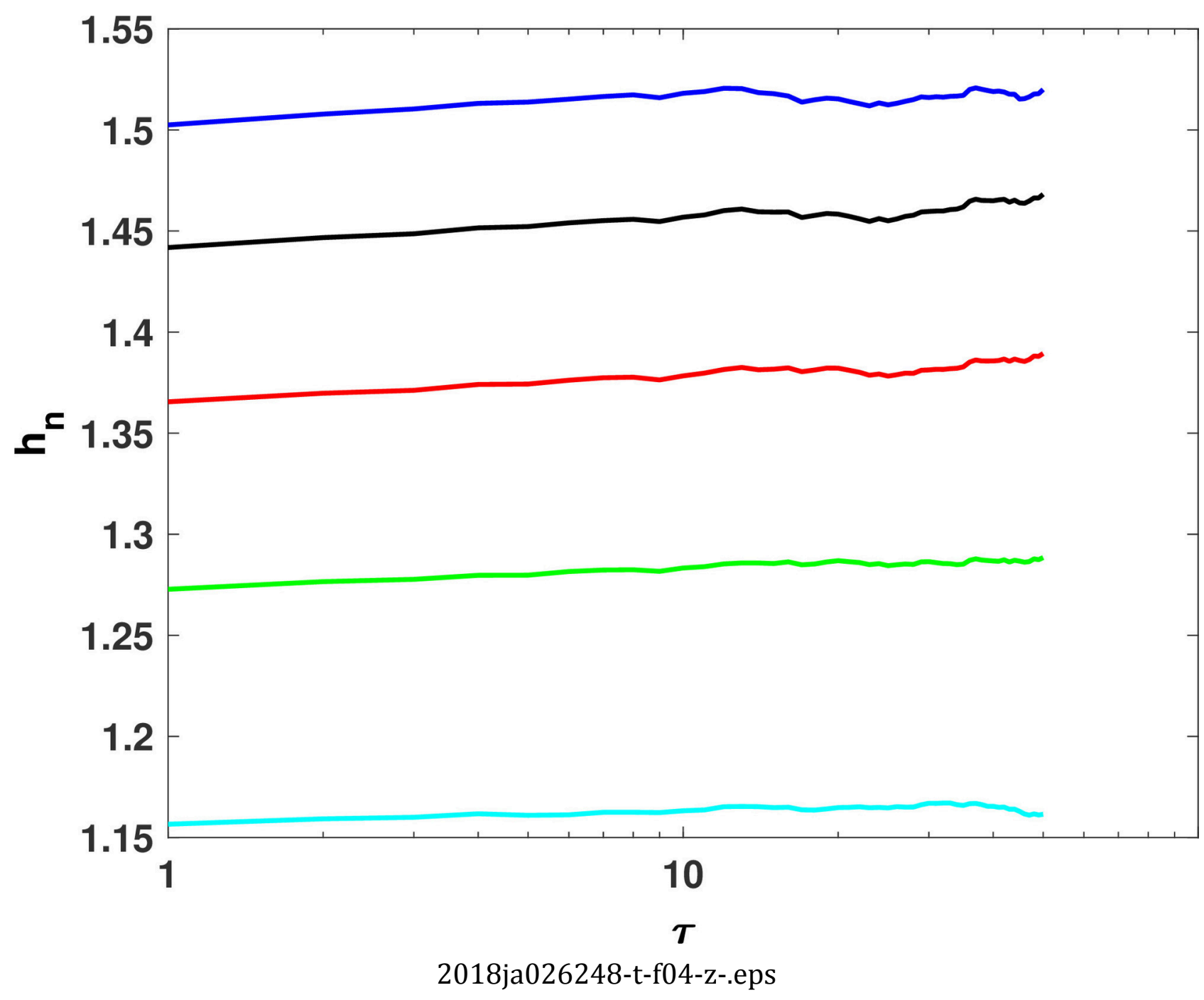

This article is protected by copyright. All rights reserved. 

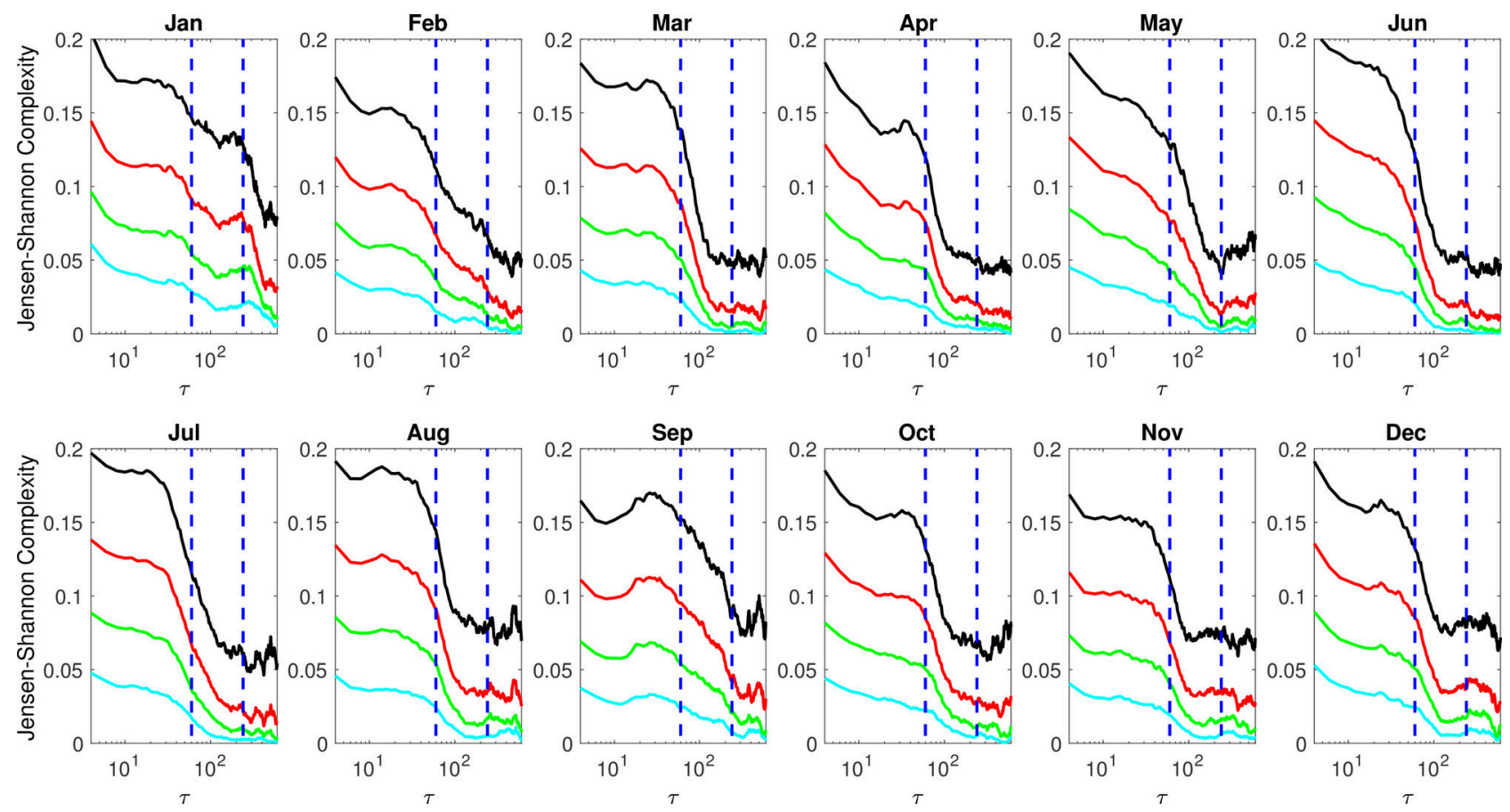

2018ja026248-t-f05-z-.eps 


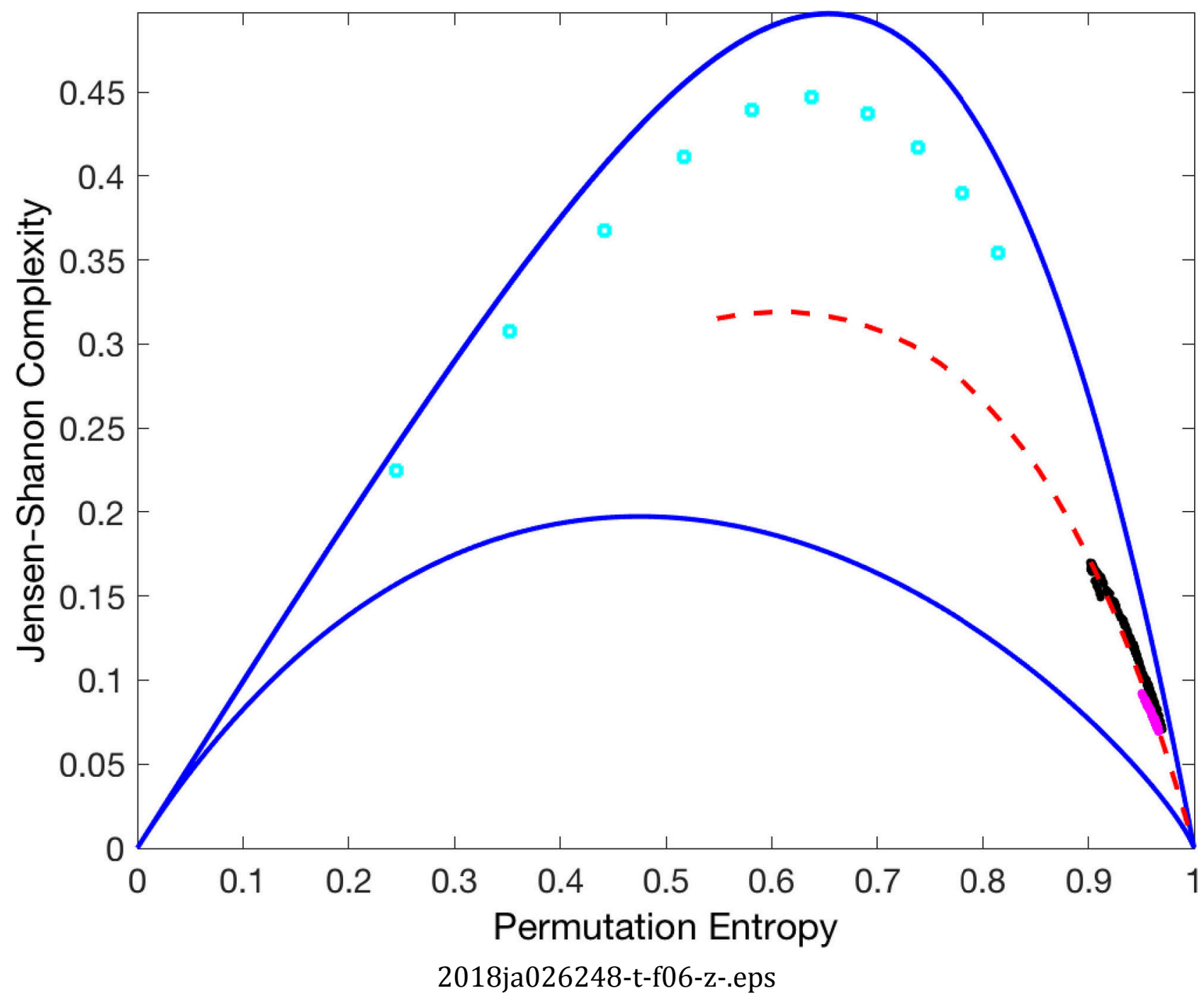

This article is protected by copyright. All rights reserved. 


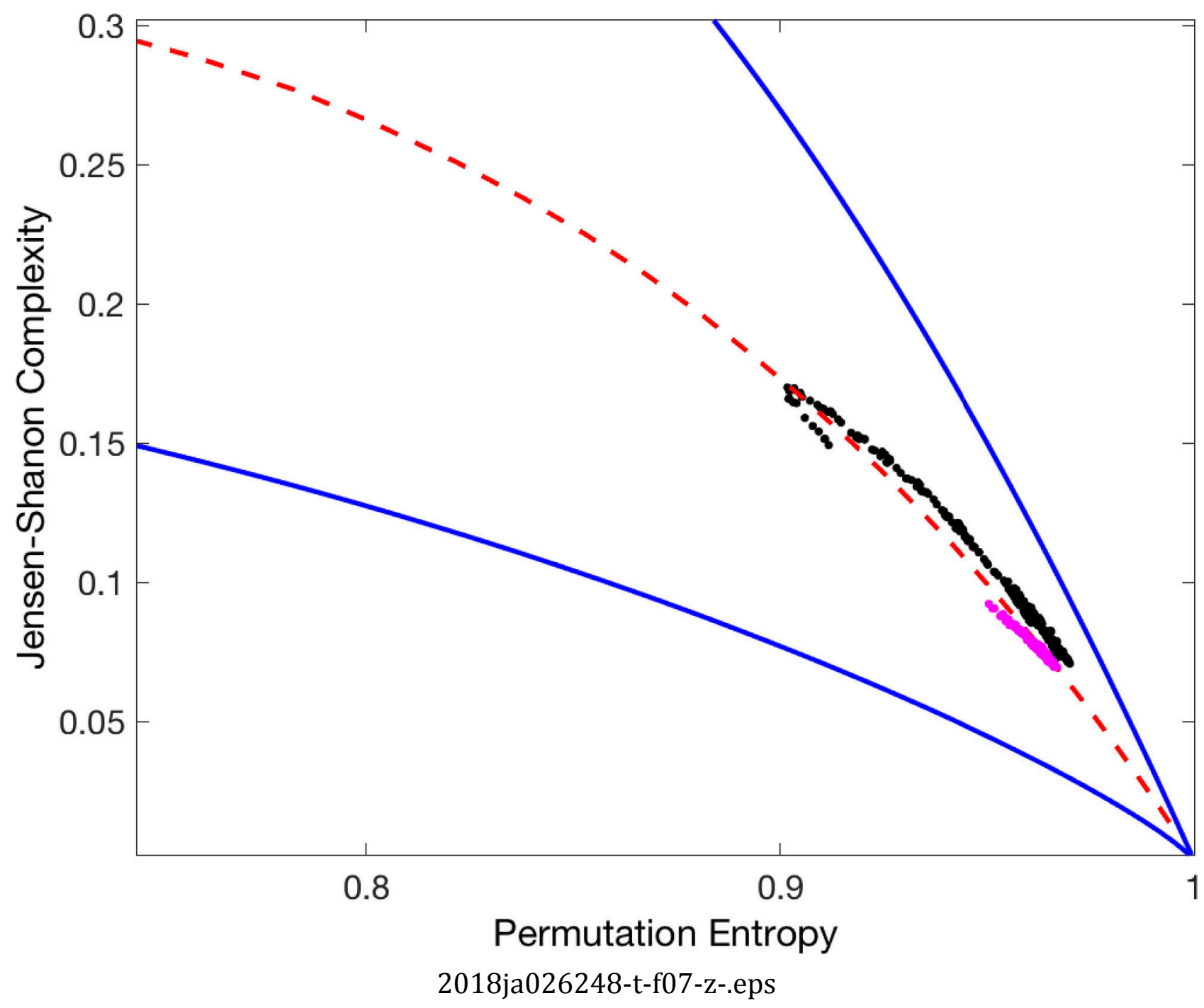

This article is protected by copyright. All rights reserved. 

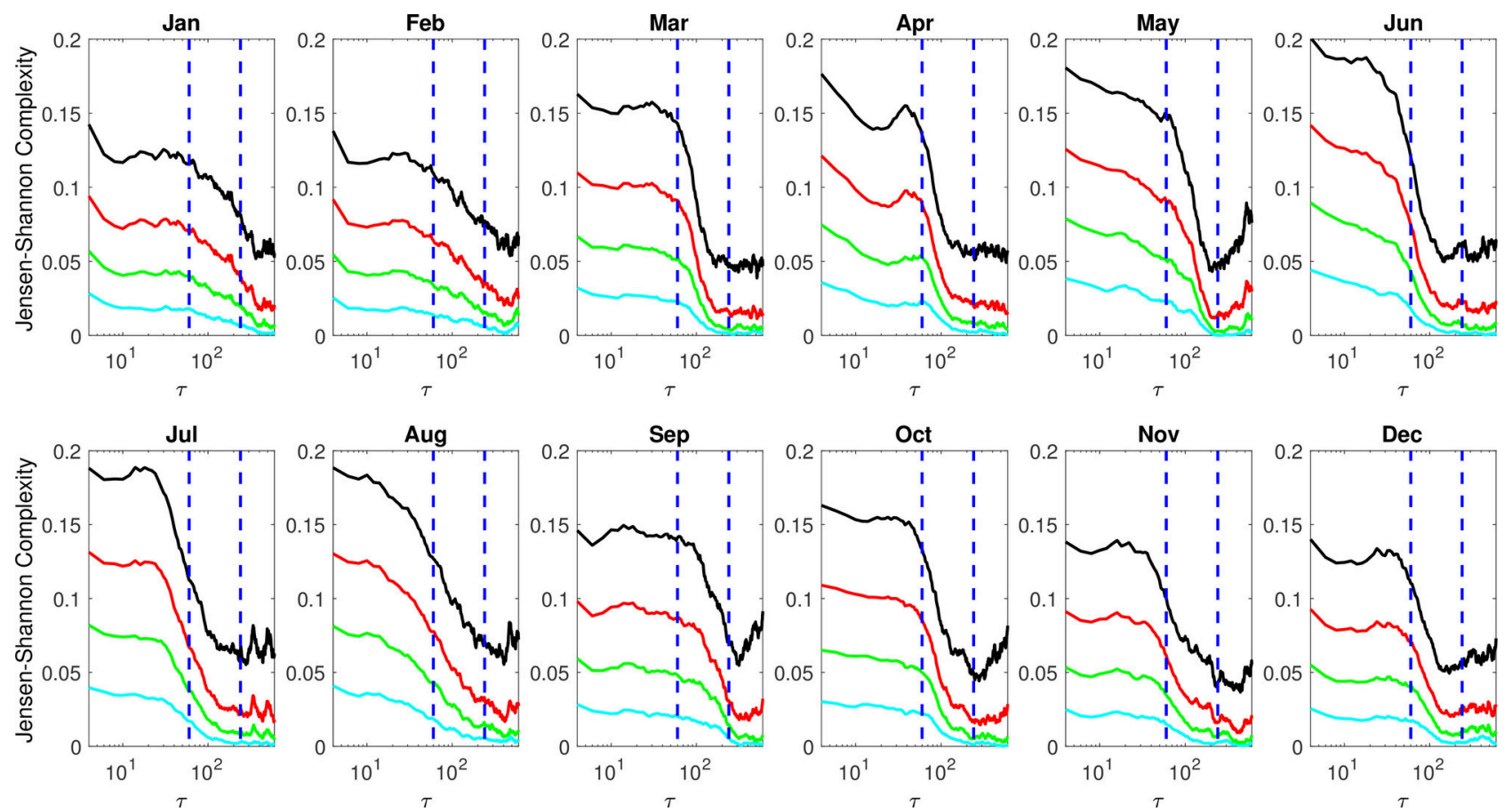

2018ja026248-t-f08-z-.eps 

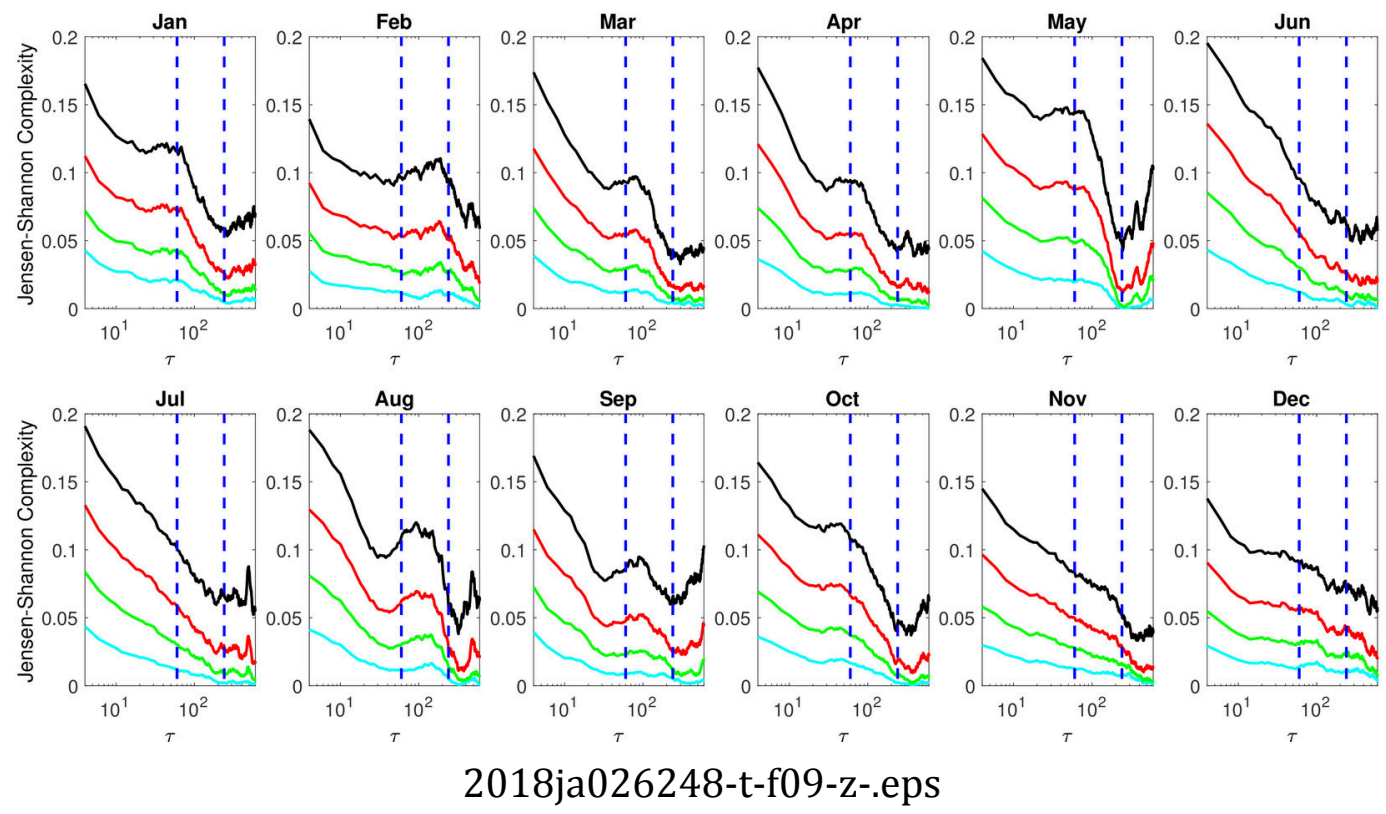

This article is protected by copyright. All rights reserved. 


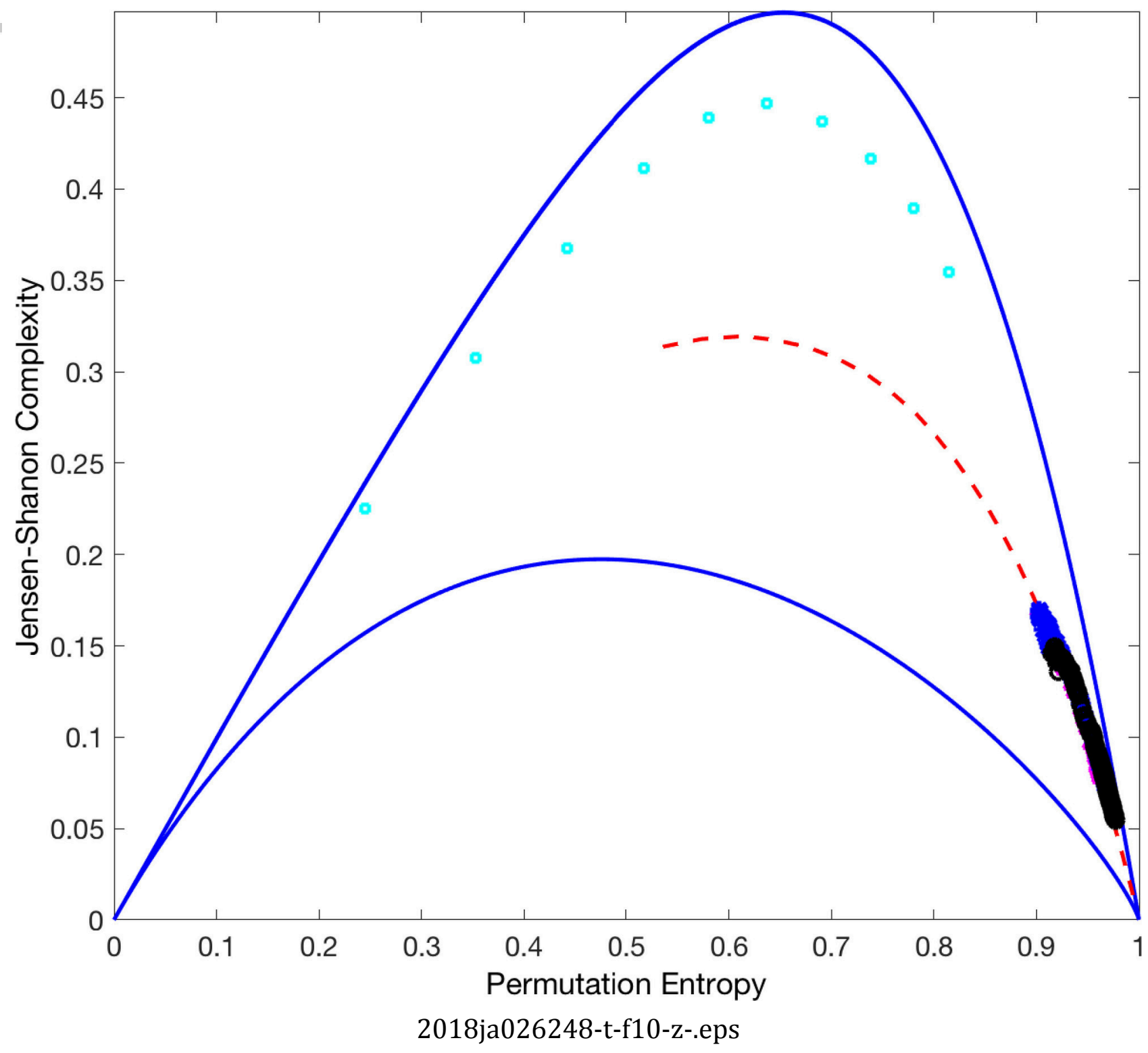

This article is protected by copyright. All rights reserved. 


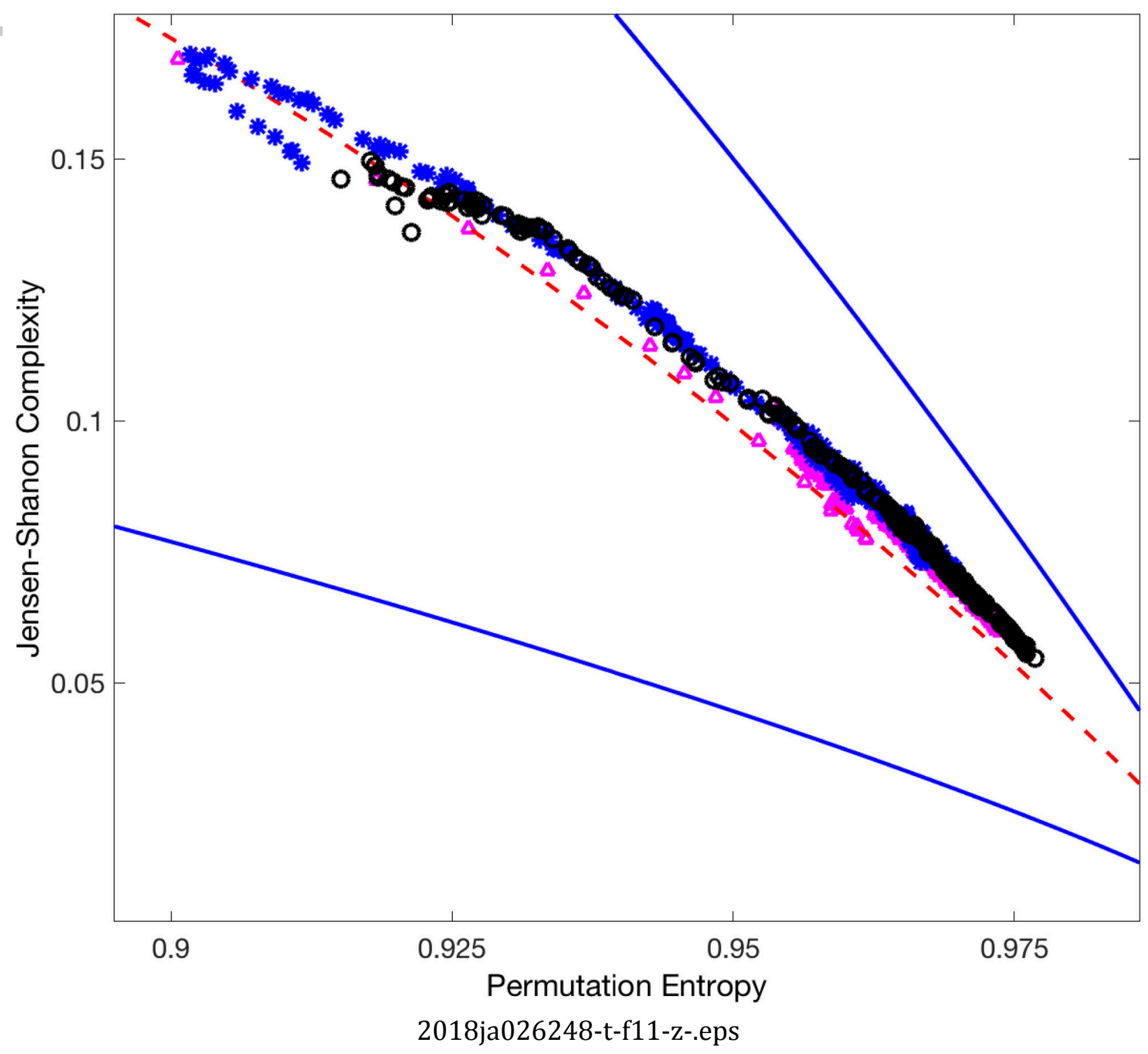

This article is protected by copyright. All rights reserved. 